Structural Assignment of a bis-Cyclopentenyl- $\beta$-cyanohydrin

Formed via Alkene Metathesis from either a Triene or a

\title{
Tetraene Precursor
}

Keith G. Andrews ${ }^{\mathrm{a}}$, Christopher S. Frampton ${ }^{\mathrm{b}}$ and Alan C. Spivey ${ }^{\mathrm{a}}$

Department of Chemistry, Imperial College London, South Kensington, London, SW7 $2 A Z$.

Pharmorphix Limited, 250 Cambridge Science Park Milton Road, Cambridge, CB4 OWE, United Kingdom

*Corresponding Author - Email: a.c.spivey@imperial.ac.uk. Tel: +44 (0)20 75945841

\section{SUPPORTING INFORMATION}

\section{Computational methods}

Predicted chemical shifts for compounds $\mathbf{9}$ and $\mathbf{1 0}$

General directions

Synthetic procedures for compounds $6,7,10$ and 12-15

NMR Spectra

References 


\section{Computational Methods}

All structures were optimised to their lowest energy gas phase conformers with the MM2 or MMFF4 forcefield. The molecules examined here have few rotatable bonds, and the possible conformers were easily identified manually. All further calculations were performed using Gaussian (version G09W; release C.01) employing the popular DFT functional, B3LYP, which has precedent for both accurate geometry optimisation and NMR shielding tensor calculation. The conformers from the initial searches were optimised using the $6-31 \mathrm{G}^{* *}$ basis set $^{3}$ for all atoms, and NMR calculations performed at the same level of theory, using the $\mathrm{GIAO}^{4}$ method. Structures were optimised in the gas phase and for unique conformers, their validity as true minima confirmed with a vibrational analysis calculation to ascertain that no imaginary modes existed. The energies were recovered with a single point energy (SPE) calculation in solution, using the CPCM (conductor-like polarisable continuum model) solvation model as implemented in Gaussian, with chloroform $\left(\mathrm{CHCl}_{3}\right)$ as the solvent. Although it is appreciated that the zero-point-energy-corrected Gibbs free energies should be used for the Boltzmann weightings, it was considered that the errors in the final MAE values using the SPE values was sufficiently suitably small for our purposes. The NMR calculations were also completed in solvent at the B3LYP/6-31G** level.

The chemical shift values for each isomer were obtained by using Boltzmann weighted average shifts over the set of conformers, calculated by first averaging the chemical shifts for degenerate symmetry related carbon environments within each conformer. Each conformer then contributed a percentage of its chemical shift value to the final total value per environment depending on its Boltzmann weighted energy contribution. A general equation is shown below, where $\sigma^{\mathrm{x}}$ is the weighted average shielding tensor of the atom(s), $\sigma_{i}^{\mathrm{x}}$ is the raw shielding tensor of the atom(s) in conformer $i$ and $E_{i}$ is the energy of the associated conformer.

$$
\sigma^{x}=\frac{\sum_{i} \sigma_{i}^{x} \exp \left(-\frac{E_{i}}{R T}\right)}{\sum_{i} \exp \left(-\frac{E_{i}}{R T}\right)}
$$

Finally, tetramethylsilane (TMS) was optimised in a symmetry restricted (tetrahedral) geometry optimisation calculation in chloroform and the single NMR shielding tensor for all carbon atoms obtained again in chloroform $\left(\mathrm{CHCl}_{3}\right)$ at the $\mathrm{B} 3 \mathrm{LYP} / 6-31 \mathrm{G}^{* *}$ level. The value obtained was $\sigma_{\mathrm{C}}=192.172$ and $\sigma_{\mathrm{H}}=31.736$. All final shifts were then calculated as $\sigma_{\mathrm{TMS}}-$ $\sigma_{\text {calc. }}$

The corrected mean absolute error (CMAE) is calculated by performing a linear regression on the experimental/predicted shifts for each isomer. The predicted shifts are then scaled using the parameters from this linear regression, according to:

$$
\delta_{\text {scaled }}=\frac{\delta_{\text {calc }}-\text { intercept }}{\text { slope }}
$$

The absolute error between each scaled shift and the corresponding experimental shift is obtained, and these values are used to calculate the mean error, which is the CMAE. 
Predicted chemical shifts for compounds 9 and 10

${ }^{1} \mathrm{H}$ predicted and experimental shifts

\begin{tabular}{|c|c|c|}
\hline \multicolumn{3}{|c|}{ DFT/B3LYP/6-31G** } \\
\hline \multicolumn{3}{|c|}{ GIAO method (CPCM, $\left.\mathrm{CHCl}_{3}\right)$} \\
\hline \multirow{2}{*}{\multicolumn{3}{|c|}{$\begin{array}{l}\text { Referenced to TMS }(\sigma=31.736) \\
\text { Experiment: } \delta_{\mathrm{H}}\left(\mathrm{CDCl}_{3}, 400 \mathrm{MHz}\right)\end{array}$}} \\
\hline & & \\
\hline & \multicolumn{2}{|c|}{${ }^{1} \mathrm{H}$ predicted shifts } \\
\hline $\begin{array}{c}\delta_{\mathrm{H}} \\
\text { Experiment }\end{array}$ & $\begin{array}{c}\delta \text { н } \\
\text { bis-cyclopentene } 10\end{array}$ & $\begin{array}{c}\delta{ }_{\text {H }} \\
\text { cis-dehydrodecalin } 9\end{array}$ \\
\hline 5.73 & 5.98 & 5.88 \\
\hline 5.69 & 5.89 & 5.83 \\
\hline 2.88 & 3.13 & 2.60 \\
\hline 2.75 & 2.85 & 2.36 \\
\hline 2.74 & 2.74 & 2.31 \\
\hline 2.46 & 2.46 & 2.29 \\
\hline 2.23 & 1.71 & 1.96 \\
\hline
\end{tabular}

${ }^{13} \mathrm{C}$ predicted and experimental shifts

\begin{tabular}{|c|c|c|}
\hline \multicolumn{3}{|c|}{ DFT/B3LYP/6-31G** } \\
\hline \multicolumn{3}{|c|}{ GIAO method (CPCM, $\left.\mathrm{CHCl}_{3}\right)$} \\
\hline \multicolumn{3}{|c|}{ Referenced to TMS $(\sigma=192.172)$} \\
\hline \multicolumn{3}{|c|}{$\begin{array}{l}\text { Experiment: } \delta_{\mathrm{c}}\left(\mathrm{CDCl}_{3}, 100 \mathrm{MHz}\right) \\
/ \mathrm{ppm}\end{array}$} \\
\hline \multicolumn{3}{|c|}{${ }^{13} \mathrm{C}$ predicted shifts } \\
\hline $\begin{array}{c}\delta{ }_{c} \\
\text { Experiment }\end{array}$ & $\begin{array}{c}\delta \delta_{\mathrm{c}} \\
\text { bis-cyclopentene } 10\end{array}$ & $\begin{array}{c}\delta \mathrm{c} \\
\text { cis-dehydrodecalin } 9\end{array}$ \\
\hline 128.48 & 126.24 & 122.56 \\
\hline 128.47 & 125.73 & 120.45 \\
\hline 125.53 & 118.89 & 117.48 \\
\hline 83.12 & 85.09 & 72.18 \\
\hline 49.24 & 52.46 & 44.14 \\
\hline 44.86 & 46.35 & 37.93 \\
\hline 41.57 & 43.51 & 35.79 \\
\hline
\end{tabular}




\section{General Directions}

All reactions were performed under an atmosphere of nitrogen and anhydrous conditions in flame or oven-dried glassware unless stated. Yields refer to chromatographically homogenous materials, unless otherwise indicated. All reagents were used as received from commercial suppliers. Anhydrous solvents were used directly following passage under nitrogen through $\mathrm{Al}_{2} \mathrm{O}_{3}$ columns in a Grubbs dry-solvent system (Innovative Technology Inc.). Flash chromatography (FC) was performed on silica gel (Merck Kieselgel 60 F254 230-400 mesh). Thin Layer Chromatography (TLC) was performed on Merck aluminium-backed plates precoated with silica $\left(0.2 \mathrm{~mm}, 60 \mathrm{~F}_{254}\right)$. Melting points were determined on a Reichardt hot stage apparatus and are uncorrected. Infra-red (IR) spectra were recorded neat on a Perkin-Elmer Paragon 1000 Fourier transform spectrometer. Only selected absorbances $\left(v_{\max }\right)$ are reported. ${ }^{1} \mathrm{H}$ NMR spectra were recorded at ambient temperature at $400 \mathrm{MHz}$ (unless stated otherwise) on a Bruker DRX-400 (or DRX-500) instrument. Chemical shifts $\left(\delta_{\mathrm{H}}\right)$ are quoted in parts per million (ppm), referenced to the residual solvent peak $\left(\mathrm{CDCl}_{3}\right.$ at $\delta_{\mathrm{H}} 7.27 \mathrm{ppm}$ and $\mathrm{MeOD}$ at $\delta_{\mathrm{H}}$ $3.35 \mathrm{ppm})$. The multiplicities of ${ }^{1} \mathrm{H}$ signals are designated by the following abbreviations: $\mathrm{s}=$ singlet; $\mathrm{d}=$ doublet; $\mathrm{dd}=$ doublet of doublets; $\mathrm{ddt}=$ doublet of doublets of triplets; $\mathrm{t}=$ triplet; $\mathrm{q}=$ quartet; $\mathrm{br}=$ board; $\mathrm{m}=$ multiplet. Coupling constants, $J$, are reported to the nearest 0.1 Hz. ${ }^{13} \mathrm{C}$ NMR spectra were recorded at $100 \mathrm{MHz}$ on a Bruker AMX-400 instrument at ambient temperature. Chemical shifts $\left(\delta_{\mathrm{C}}\right)$ are quoted in ppm referenced to $\left(\mathrm{CDCl}_{3}\right.$ at $\delta_{\mathrm{C}} 77.1$ ppm). Low resolution mass spectra $(\mathrm{m} / \mathrm{z})$ were recorded on either a VG platform II or VG AutoSpec spectrometers; molecular ions $\left(\mathrm{M}^{+}, \mathrm{MH}^{+}, \mathrm{MNH}_{4}^{+}\right)$are reported. High Resolution Mass Spectrometry (HRMS) measurements are valid to $\pm 5.2 \mathrm{ppm}$.

Ethyl 2-allyl-2-cyanopent-4-enoate $12^{\underline{5}}$

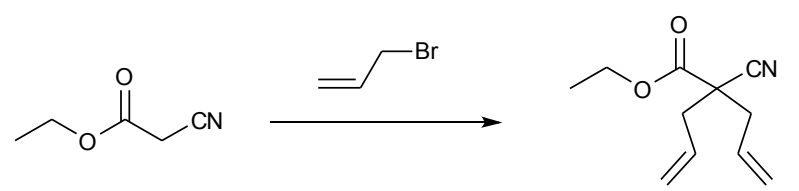

To a stirred solution of ethyl cyanoacetate $(500 \mathrm{mg}, 4.42 \mathrm{mmol})$ in DMF $(20 \mathrm{~mL})$ was added allyl bromide $(1.12 \mathrm{~g}, 9.28 \mathrm{mmol})$ and caesium carbonate $(2.88 \mathrm{~g}, 8.84 \mathrm{mmol})$. The reaction mixture was stirred at $0{ }^{\circ} \mathrm{C}$ for 15 min warming to $\mathrm{RT}$ over $3 \mathrm{~h}$. The reaction mixture was concentrated in vacuo and the crude residue partitioned between sat. aqueous sodium hydrogen carbonate solution $(10 \mathrm{~mL})$ and ethyl acetate $(40 \mathrm{~mL})$ and extracted with further ethyl acetate $(2 \times 40 \mathrm{~mL})$. The combined organics were washed with sat. aqueous sodium chloride solution $(3 \times 40 \mathrm{~mL})$ and dried over magnesium sulfate. The crude residue was purified by flash column chromatography eluting with 1:9 EtOAc:pet ether to give diene $\mathbf{1 2}$ as a colourless oil (840 mg, 98\%). IR (neat) $2246(\mathrm{CN})$, 1741 $(\mathrm{C}=\mathrm{O}), 1644(\mathrm{C}=\mathrm{C}) \mathrm{cm}^{-1} .{ }^{1} \mathrm{H}$ NMR $\left(\mathrm{CDCl}_{3}, 400 \mathrm{MHz}\right): \delta 5.84(\mathrm{ddt}, J=17.1,9.8,7.3$ $\mathrm{Hz}, 2 \mathrm{H},=\mathrm{CH}), 5.32-5.21\left(\mathrm{~m}, 4 \mathrm{H}, \mathrm{CH}_{2}\right), 4.28\left(\mathrm{q}, J=7.1 \mathrm{~Hz}, 2 \mathrm{H}, \mathrm{OCH}_{2} \mathrm{CH}_{3}\right), 2.68(\mathrm{dd}, J$ $=14.0,7.3 \mathrm{~Hz}, 2 \mathrm{H}, 2 \times \mathrm{CHH}), 2.58(\mathrm{dd}, J=14.0,7.3 \mathrm{~Hz}, 2 \mathrm{H}, 2 \times \mathrm{CH} H), 1.33(\mathrm{t}, J=7.1$ $\left.\mathrm{Hz}, 3 \mathrm{H}, \mathrm{OCH}_{2} \mathrm{CH}_{3}\right) \mathrm{ppm} ;{ }^{13} \mathrm{C} \mathrm{NMR}\left(\mathrm{CDCl}_{3}, 100 \mathrm{MHz}\right): \delta 168.0,130.5,121.0,118.5,62.8$, 49.3, 40.7, $14.2 \mathrm{ppm} ; \mathrm{MS}\left(\mathrm{CI}^{+}\right) \mathrm{m} / z 211\left(\mathrm{MNH}_{4}{ }^{+}\right)$; HRMS $\left(\mathrm{CI}^{+}\right)$calcd. for $\mathrm{C}_{11} \mathrm{H}_{19} \mathrm{~N}_{2} \mathrm{O}_{2}$ $\left(\mathrm{MNH}_{4}{ }^{+}\right) ; 211.1447$, found 211.1453, $\Delta=2.8 \mathrm{ppm}$. 


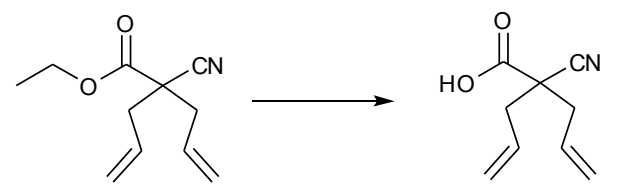

Ester $12(3170 \mathrm{mg}, 16.4 \mathrm{mmol})$ was dissolved in ethanol $(20 \mathrm{~mL})$ and cooled to $0{ }^{\circ} \mathrm{C}$. Separately, potassium tert-butoxide $(2760 \mathrm{mg}, 24.6 \mathrm{mmol})$ was dissolved in water (10 $\mathrm{mL}$ ), in an exothermic reaction, and the resulting solution was added dropwise to the stirred ethanol solution. After 15-30 min, the reaction mixture was concentrated in vacuo. The resulting residue was diluted with $1 \mathrm{M} \mathrm{NaOH}$ solution, extracted with diethyl ether (5

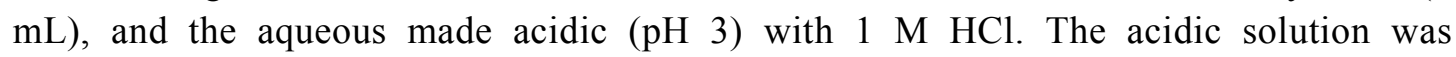
extracted with ethyl acetate $(6 \times 50 \mathrm{~mL})$ and the combined organic phases dried and concentrated to give acid $\mathbf{1 3}$ as a white crystalline solid (2.5 g, 74\%). IR (neat) $\mathrm{v}$ max 2253 $(\mathrm{CN}), 1727(\mathrm{C}=\mathrm{O}), 1645(\mathrm{C}=\mathrm{C}), 1420(\mathrm{OH}) \mathrm{cm}^{-1} ;{ }^{1} \mathrm{H}$ NMR $\left(400 \mathrm{MHz}\right.$, Methanol- $\left.d_{4}\right) \delta$ $5.86(\mathrm{ddt}, J=17.0,10.0,7.3 \mathrm{~Hz}, 2 \mathrm{H}, 2 \times \mathrm{CH}), 5.27(\mathrm{dd}, J=17.0,1.6 \mathrm{~Hz}, 2 \mathrm{H}, 2 \times=$ $\mathrm{C} H \mathrm{H}), 5.26(\mathrm{dd}, J=10.0,1.6 \mathrm{~Hz}, 2 \mathrm{H}, 2 \times=\mathrm{CH} H), 2.67(\mathrm{dd}, J=13.9,7.3 \mathrm{~Hz}, 2 \mathrm{H}, 2 \times$ $\mathrm{C} H \mathrm{H}), 2.58(\mathrm{dd}, J=13.9,7.3 \mathrm{~Hz}, 2 \mathrm{H}, 2 \times \mathrm{CH} H) \mathrm{ppm} ;{ }^{13} \mathrm{C}$ NMR (methanol- $\left.d_{4}, 100 \mathrm{MHz}\right)$ : $\delta$ 169.4, 131.0, 119.5, 118.6, 49.8, $40.3 \mathrm{ppm} ; \mathrm{MS}\left(\mathrm{CI}^{+}\right) \mathrm{m} / z 183\left(\mathrm{MNH}_{4}{ }^{+}\right)$; HRMS $\left(\mathrm{CI}^{+}\right)$ calcd. for $\mathrm{C}_{9} \mathrm{H}_{15} \mathrm{~N}_{2} \mathrm{O}_{2}\left(\mathrm{MNH}_{4}{ }^{+}\right) ; 183.1134$, found 183.1127, $\Delta=-3.8 \mathrm{ppm}$.

\section{2,2,3-Triallyl-3-hydroxyhex-5-enenitrile 6}

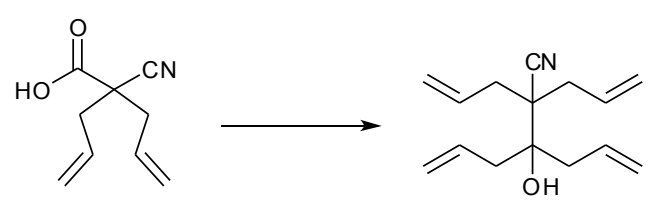

Dry magnesium turnings ( $824 \mathrm{mg}, 33.92 \mathrm{mmol}$ ) were sonicated before covering in THF and refluxing briefly with iodine $(20.3 \mathrm{mg}, 0.08 \mathrm{mmol})$. A few drops of allyl bromide were added, and the reaction heated gently until the yellow colour disappeared. A solution of the remaining allyl bromide $(2565 \mathrm{mg}, 21.2 \mathrm{mmol})$ in tetrahydrofuran $(20 \mathrm{~mL})$ was added dropwise over $1 \mathrm{~h}$ and stirred for a further $2 \mathrm{~h}$ at RT. Meanwhile, acid 13 (1400 $\mathrm{mg}, 8.48 \mathrm{mmol})$ was dissolved in $\mathrm{CH}_{2} \mathrm{Cl}_{2}(35 \mathrm{~mL})$ and stirred at $0{ }^{\circ} \mathrm{C}$ during cautious addition of oxalyl chloride (1399 $\mathrm{mg}, 11.02 \mathrm{mmol}$ ). Two drops of DMF were added and the reaction mixture stirred at RT for $3 \mathrm{~h}$. After this time, the solvent was evaporated in vacuo, and the resulting residue redissolved in THF $(5 \mathrm{~mL})$. To this solution was added the Grignard reagent solution formed above, dropwise at $0{ }^{\circ} \mathrm{C}$. After $3 \mathrm{~h}$ stirring at RT, the reaction mixture was quenched with sat. aqueous ammonium chloride solution (40 $\mathrm{mL})$ and extracted with $\mathrm{CH}_{2} \mathrm{Cl}_{2}(3 \times 50 \mathrm{~mL})$. The combined organic layers were washed with aqueous $\mathrm{HCl}$ and brine, dried over sodium sulfate, filtered, concentrated, and purified by flash column chromatography eluting with 15:1 pet ether:EtOAc to give tetraene 6 as a pale yellow oil $(120 \mathrm{mg}, 9 \%)$. IR (neat) $v \max 3471(\mathrm{OH}), 3079,2233$ $(\mathrm{CN}), 1639(\mathrm{C}=\mathrm{C}) \mathrm{cm}^{-1} ;{ }^{1} \mathrm{H} \mathrm{NMR}\left(\mathrm{CDCl}_{3}, 400 \mathrm{MHz}\right): \delta 6.07-5.88(\mathrm{~m}, 4 \mathrm{H}, 4 \times=\mathrm{CH})$, $5.28-5.19\left(\mathrm{~m}, 8 \mathrm{H}, 4 \times=\mathrm{CH}_{2}\right), 2.70-2.62(\mathrm{~m}, 2 \mathrm{H}, 2 \times \mathrm{CHH}), 2.55(\mathrm{~d}, J=7.3 \mathrm{~Hz}, 4 \mathrm{H}, 2$ $\left.\times \mathrm{CH}_{2}\right), 2.50(\mathrm{dd}, J=14.4,7.8 \mathrm{~Hz}, 2 \mathrm{H}, 2 \times \mathrm{CH} H), 2.15(\mathrm{~s}, 1 \mathrm{H}) \mathrm{ppm} ;{ }^{13} \mathrm{C} \mathrm{NMR}\left(\mathrm{CDCl}_{3}\right.$, $100 \mathrm{MHz}): \delta 133.5,132.9,121.6,120.0,119.4,75.9,50.4,41.3,37.3 \mathrm{ppm} ; \mathrm{MS}\left(\mathrm{CI}^{+}\right) \mathrm{m} / \mathrm{z}$ 
$249\left(\mathrm{MNH}_{4}^{+}\right)$; HRMS $\left(\mathrm{CI}^{+}\right)$calcd. for $\mathrm{C}_{15} \mathrm{H}_{25} \mathrm{~N}_{2} \mathrm{O}\left(\mathrm{MNH}_{4}^{+}\right) ; 249.1967$, found 249.1966, $\Delta$ $=-0.4 \mathrm{ppm}$.

\section{Ethyl 1-cyanocyclopent-3-ene-1-carboxylate 14}

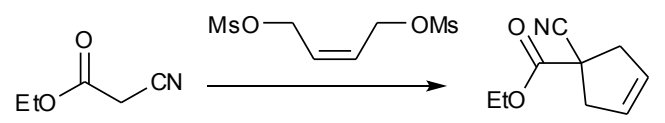

Ethyl cyanoacetate $(47.0 \mu \mathrm{L}, 0.44 \mathrm{mmol})$ and (Z)-but-2-en-1,4-diol dimethanesulfonate ${ }^{7}$ (73.1 $\mathrm{mg}, 0.40 \mathrm{mmol})$ were added to caesium carbonate $(260 \mathrm{mg}, 0.80 \mathrm{mmol})$ in DMF (10 $\mathrm{mL}$ ) and the reaction mixture stirred for $30 \mathrm{~min}$ without restricting the temperature rise. Sat. aqueous sodium hydrogen carbonate solution $(100 \mathrm{~mL})$ was added to the reaction mixture before extracting with ethyl acetate $(4 \times 50 \mathrm{~mL})$. The combined organic layers were washed with sat. aqueous sodium chloride solution $(3 \times 20 \mathrm{~mL})$, dried over sodium sulfate and concentrated in vacuo. The crude residue was purified by flash column chromatography eluting with 1:10 EtOAc:pet ether to give the cyclopentene $\mathbf{1 4}$ as a colourless oil $(55.4 \mathrm{mg}, 84 \%)$. IR (neat) $2246(\mathrm{CN}), 1739(\mathrm{C}=\mathrm{O}) \mathrm{cm}^{-1} ;{ }^{1} \mathrm{H} \mathrm{NMR}\left(\mathrm{CDCl}_{3}\right.$, $400 \mathrm{MHz}): \delta 5.72(\mathrm{~s}, 2 \mathrm{H}, \mathrm{HC}=\mathrm{CH}), 4.31\left(\mathrm{q}, J=7.1 \mathrm{~Hz}, 1 \mathrm{H}, \mathrm{OCH}_{2} \mathrm{CH}_{3}\right), 3.15\left(\mathrm{~m},{ }^{2} J=\right.$ $15.4 \mathrm{~Hz}, 1 \mathrm{H}, \mathrm{CHH}), 3.09\left(\mathrm{~m},{ }^{2} J=15.4 \mathrm{~Hz}, 1 \mathrm{H}, \mathrm{CH} H\right), 1.36\left(\mathrm{t}, J=7.2 \mathrm{~Hz}, 2 \mathrm{H}, \mathrm{OCH}_{2} \mathrm{CH}_{3}\right)$ ppm; ${ }^{13} \mathrm{C}$ NMR $\left(\mathrm{CDCl}_{3}, 400 \mathrm{MHz}\right): \delta 169.2,127.5,121.2,63.1,45.4,44.0,14.0 \mathrm{ppm}$; $\mathrm{MS}\left(\mathrm{CI}^{+}\right) \mathrm{m} / z 183\left(\mathrm{MNH}_{4}{ }^{+}\right)$; HRMS $\left(\mathrm{CI}^{+}\right)$calcd. for $\mathrm{C}_{9} \mathrm{H}_{15} \mathrm{~N}_{2} \mathrm{O}_{2}\left(\mathrm{MNH}_{4}{ }^{+}\right) ; 183.1134$, found $183.1143, \Delta=4.9 \mathrm{ppm}$.

\section{1-Cyanocyclopent-3-ene-1-carboxylic acid 15}

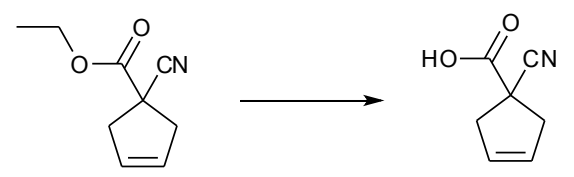

To a solution of ester $14(40 \mathrm{mg}, 0.24 \mathrm{mmol})$ in ethanol $(4 \mathrm{~mL})$ was added a solution of potassium tert-butoxide $(29.2 \mathrm{mg}, 0.26 \mathrm{mmol})$ in water $(1 \mathrm{~mL})$. The reaction mixture was stirred at RT for $1 \mathrm{~h}$ before being concentrated to an oil, redissolved in dilute sodium hydroxide $(5 \mathrm{~mL})$ and extracted once with diethyl ether $(1 \mathrm{~mL})$. The aqueous phase was acidified to $\mathrm{pH} 2$ and extracted with ethyl acetate $(4 \times 20 \mathrm{~mL})$. The combined organic layers were dried and concentrated to give acid 15 as a white powder $(29.3 \mathrm{mg}, 90 \%)$. IR (neat) $v \max 2252(\mathrm{CN}), 1721(\mathrm{C}=\mathrm{O}) \mathrm{cm}^{-1} ;{ }^{1} \mathrm{H} \mathrm{NMR}\left(400 \mathrm{MHz}\right.$, Methanol- $\left.d_{4}\right) \delta 5.72(\mathrm{~s}, 2 \mathrm{H}$, $\mathrm{HC}=\mathrm{CH}), 3.15\left(\mathrm{~m},{ }^{2} \mathrm{~J}=14.8 \mathrm{~Hz}, 2 \mathrm{H}, 2 \times \mathrm{CHH}\right), 2.97\left(\mathrm{~m},{ }^{2} \mathrm{~J}=14.8 \mathrm{~Hz}, 2 \mathrm{H}, 2 \times \mathrm{CH} H\right)$ ppm; ${ }^{13} \mathrm{C}$ NMR (400 MHz, Methanol- $\left.d_{4}\right) \delta 172.4,127.4,123.1,47.1,44.0$ ppm; MS(CI+) $m / z 155\left(\mathrm{MNH}_{4}^{+}\right)$; HRMS $\left(\mathrm{CI}^{+}\right)$calcd. for $\mathrm{C}_{7} \mathrm{H}_{11} \mathrm{~N}_{2} \mathrm{O}_{2}\left(\mathrm{MNH}_{4}^{+}\right)$; 155.0821, found $155.0826, \Delta=3.2 \mathrm{ppm}$. 


\section{1-(4-Hydroxyhepta-1,6-dien-4-yl)cyclopent-3-ene-1-carbonitrile 7}<smiles>C=CCC1(O)CC=CC1(C)CC=CC</smiles>

Dry magnesium turnings $(306 \mathrm{mg}, 12.6 \mathrm{mmol}$ ) were sonicated before covering in THF and refluxing briefly with iodine $(20.3 \mathrm{mg}, 0.08 \mathrm{mmol})$. Allyl bromide $(762 \mathrm{mg}, 6.3$ mmol) was dropped in slowly over 90 min while stirring rapidly at RT. Meanwhile, acid $15(288 \mathrm{mg}, 2.1 \mathrm{mmol})$ was dissolved in $\mathrm{CH}_{2} \mathrm{Cl}_{2}(6 \mathrm{~mL})$ and stirred at $0{ }^{\circ} \mathrm{C}$ during cautious addition of a solution of oxalyl chloride $(216 \mu \mathrm{L}, 2.52 \mathrm{mmol})$ in $\mathrm{CH}_{2} \mathrm{Cl}_{2}(1 \mathrm{~mL})$. A drop of DMF was added and the reaction mixture was heated at reflux for $3 \mathrm{~h}$. After this time, the solvent was evaporated in vacuo and the resulting yellow oil redissolved in THF ( $3 \mathrm{~mL}$ ). To this was added the Grignard reagent solution formed above, dropwise at $0{ }^{\circ} \mathrm{C}$. After $3 \mathrm{~h}$ stirring at $\mathrm{RT}$, the reaction mixture was quenched with sat. aqueous ammonium chloride solution $(40 \mathrm{~mL})$ and extracted with $\mathrm{CH}_{2} \mathrm{Cl}_{2}(3 \times 50 \mathrm{~mL})$. The combined organic layers were washed with aqueous $\mathrm{HCl}$ and brine, dried over sodium sulfate, filtered, concentrated and purified by flash column chromatography eluting with 15:1 pet ether:EtOAc to give triene 7 as a pale yellow oil. IR (neat) $v \max 3467(\mathrm{OH})$, $2235(\mathrm{CN}), 1640(\mathrm{C}=\mathrm{C}) \mathrm{cm}^{-1} ;{ }^{1} \mathrm{H} \mathrm{NMR}\left(\mathrm{CDCl}_{3}, 400 \mathrm{MHz}\right): \delta 5.95(\mathrm{ddt}, J=17.6,10.3,7.4$ $\mathrm{Hz}, 2 \mathrm{H}, 2 \times=\mathrm{CH}), 5.71(\mathrm{~s}, 2 \mathrm{H}, \mathrm{HC}=\mathrm{CH}), 5.31-5.16\left(\mathrm{~m}, 4 \mathrm{H}, 2 \times=\mathrm{CH}_{2}\right), 2.92(\mathrm{~d}, J=14.8$ $\mathrm{Hz}, 2 \mathrm{H}, 2 \times \mathrm{CHH}), 2.78(\mathrm{~d}, J=14.8 \mathrm{~Hz}, 2 \mathrm{H}, 2 \times \mathrm{CH} H), 2.58(\mathrm{dd}, J=14.1,7.5 \mathrm{~Hz}, 2 \mathrm{H}, 2$ $\times \mathrm{CHH}), 2.47(\mathrm{dd}, J=14.1,7.5 \mathrm{~Hz}, 2 \mathrm{H}, 2 \times \mathrm{CH} H), 2.00(\mathrm{~s}, 1 \mathrm{H}) \mathrm{ppm} ;{ }^{13} \mathrm{C} \mathrm{NMR}\left(\mathrm{CDCl}_{3}\right.$, $100 \mathrm{MHz}): \delta 132.8,128.2,125.3,120.2,74.2,51.5,41.8,40.7 \mathrm{ppm} ; \mathrm{MS}\left(\mathrm{CI}^{+}\right) \mathrm{m} / z 221$ $\left(\mathrm{MNH}_{4}^{+}\right)$; HRMS $\left(\mathrm{CI}^{+}\right)$calcd. for $\mathrm{C}_{13} \mathrm{H}_{21} \mathrm{~N}_{2} \mathrm{O}\left(\mathrm{MNH}_{4}^{+}\right)$; 221.1654, found 221.1647, $\Delta=$ $-3.2 \mathrm{ppm}$.

\section{1'-Hydroxy-[1,1'-bi(cyclopentane)]-3,3'-diene-1-carbonitrile 10}

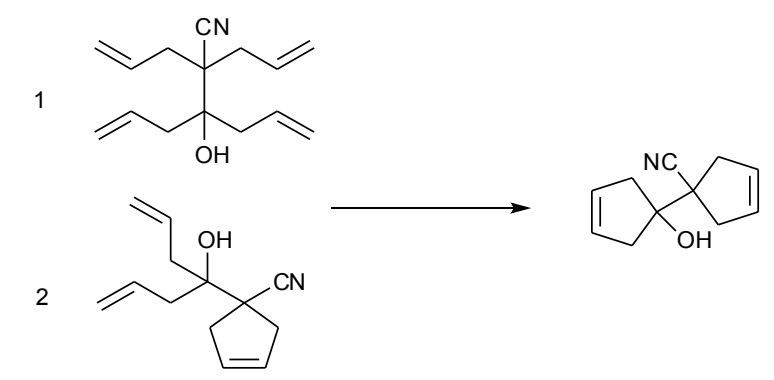

Method 1 - from tetraene 6: To a solution of tetraene $6(80 \mathrm{mg}, 0.35 \mathrm{mmol})$ in $\mathrm{CH}_{2} \mathrm{Cl}_{2}(15$ $\mathrm{mL})$ was added Grubbs II catalyst \{Trnka, $2000 \# 708\}$ (17.0 mg, $0.02 \mathrm{mmol})$ and the resulting reaction mixture stirred at RT for $14 \mathrm{~h}$. After concentration in vacuo, analysis of the ${ }^{1} \mathrm{H}$ NMR spectrum of the crude residue revealed the formation of two products in a ratio of 2:1. The major product corresponds to bis-cyclopentene $\mathbf{1 0}$ (see below) and the minor product corresponds to the known trans-2,3,6,7-dehydrodecalin 8. \{Webber, $2013 \# 78\}$

Method 2 - from triene 7: To a solution of triene 7 (300 mg, $1.48 \mathrm{mmol})$ in $\mathrm{CH}_{2} \mathrm{Cl}_{2}(30 \mathrm{~mL})$ at $0{ }^{\circ} \mathrm{C}$ was added Grubbs II catalyst $\{$ Trnka, $2000 \# 708\}$ (59.4 mg, $\left.0.07 \mathrm{mmol}\right)$ and the resulting 
reaction mixture stirred at RT for $18 \mathrm{~h}$. After concentration in vacuo, analysis of the ${ }^{1} \mathrm{H}$ NMR spectrum of the crude residue revealed the formation of a single product. Purification by flash column chromatography eluting with 1:11 EtOAc:pet ether gave bis-cyclopentene $\mathbf{1 0}$ as a white crystalline solid (190 mg, 73\%). Mp. 54.5-56.0 ${ }^{\circ} \mathrm{C}$; IR (neat) $v_{\text {max }} 3461(\mathrm{OH}), 3060$, 2925, 2860, 2237 (CN), 1622 (C=C), 1431, 1368, $1340 \mathrm{~cm}^{-1} ;{ }^{1} \mathrm{H}$ NMR $\left(\mathrm{CDCl}_{3}, 400 \mathrm{MHz}\right): \delta$ $5.73(\mathrm{~m}, 2 \mathrm{H}, \mathrm{HC}=\mathrm{CH}), 5.69(\mathrm{~m}, 2 \mathrm{H}, \mathrm{HC}=\mathrm{CH}), 2.97-2.86(\mathrm{~m}, 2 \mathrm{H}, 2 \times \mathrm{CHH}), 2.83-2.71(\mathrm{~m}$, $\left.4 \mathrm{H}, 2 \times \mathrm{CH}_{2}\right), 2.53-2.42(\mathrm{~m}, 2 \mathrm{H}, 2 \times \mathrm{CH} H), 2.08(\mathrm{br}, \mathrm{s}, 1 \mathrm{H}, \mathrm{OH}) \mathrm{ppm} ;{ }^{1} \mathrm{H}$ NMR (acetone- $d_{6}$, $500 \mathrm{MHz}) \delta 5.70(\mathrm{~m}, 2 \mathrm{H}, \mathrm{HC}=\mathrm{CH}), 5.68(\mathrm{~m}, 2 \mathrm{H}, \mathrm{HC}=\mathrm{CH}), 4.43(\mathrm{~s}, 1 \mathrm{H}, \mathrm{OH}), 2.83-2.66(\mathrm{~m}$, $\left.6 \mathrm{H}, \mathrm{CH}_{2}\right), 2.51-2.41\left(\mathrm{~m}, 2 \mathrm{H}, \mathrm{CH}_{2}\right) .{ }^{1} \mathrm{H}-{ }^{1} \mathrm{H}$ TOCSY NMR (acetone- $d_{6}, 500 \mathrm{MHz}$ ): no crosspeak between alkene proton environments at $\delta 5.70$ and $5.68 ;{ }^{13} \mathrm{C} \mathrm{NMR}\left(\mathrm{CDCl}_{3}, 100 \mathrm{MHz}\right): \delta$ 128.48-128.47 (overlapping signals), 125.3, 83.1, 49.2, 44.9, $41.6 \mathrm{ppm} ; \mathrm{MS}\left(\mathrm{CI}^{+}\right) \mathrm{m} / z 193$ $\left(\mathrm{MNH}_{4}^{+}\right)$; HRMS $\left(\mathrm{CI}^{+}\right)$calcd. for $\mathrm{C}_{11} \mathrm{H}_{17} \mathrm{~N}_{2} \mathrm{O}\left(\mathrm{MNH}_{4}^{+}\right)$; 193.1341, found 193.1331, $\Delta=-5.2$ ppm. Further re-crystallisation of a portion of this product via the vapour diffusion method using EtOAc:pentane gave white needles suitable for X-ray crystallography. 
Crystal data for 10: $\mathrm{C}_{11} \mathrm{H}_{13} \mathrm{NO}$.

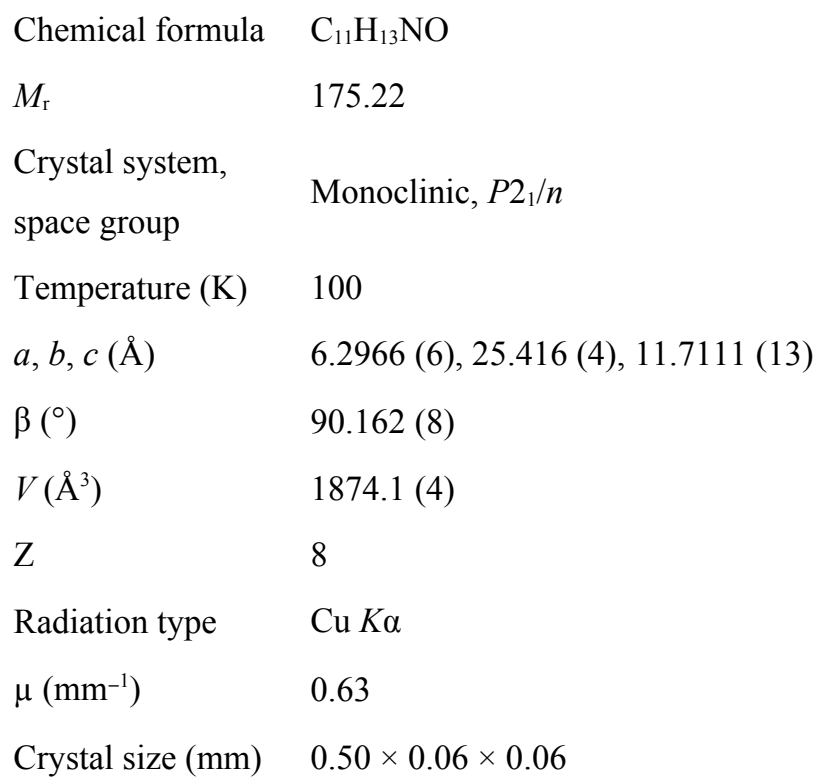

Refinement

$R\left[F^{2}>2 \sigma\left(F^{2}\right)\right]$,

$w R\left(F^{2}\right), S$

No. of reflections 3793

No. of parameters 281

No. of restraints 0 
$\mathrm{H}$-atom treatment $\mathrm{H}$ atoms treated by a mixture of independent and constrained refinement $\Delta \rho_{\max }, \Delta \rho_{\min }\left(\mathrm{e} \AA^{-3}\right) \quad 0.30,-0.21$

Computer programs: CrysAlis PRO, Agilent Technologies, Version 1.171.35.19 (release 27-10-2011 CrysAlis171 .NET) (compiled Oct 27 2011,15:02:11), SHELXTL (Sheldrick, 2008), Mercury 2.4 (Macrae et al., 1998). 
Table 1 Selected geometric parameters $\left(\AA,{ }^{\circ}\right)$

$\begin{array}{llll}\text { O1A-C6A } & 1.4270(17) & \mathrm{C} 1 \mathrm{~B}-\mathrm{C} 6 \mathrm{~B} & 1.545(5) \\ \mathrm{N} 1 \mathrm{~A}-\mathrm{C} 11 \mathrm{~A} & 1.146(2) & \mathrm{C} 1 \mathrm{~B}-\mathrm{C} 5 \mathrm{~B} & 1.556(3) \\ \mathrm{C} 1 \mathrm{~A}-\mathrm{C} 11 \mathrm{~A} & 1.4693(18) & \mathrm{C} 1 \mathrm{~B}-\mathrm{C} 2 \mathrm{~B} & 1.581(3) \\ \mathrm{C} 1 \mathrm{~A}-\mathrm{C} 5 \mathrm{~A} & 1.5543(19) & \mathrm{C} 2 \mathrm{~B}-\mathrm{C} 3 \mathrm{~B} & 1.501(2) \\ \mathrm{C} 1 \mathrm{~A}-\mathrm{C} 6 \mathrm{~A} & 1.5579(19) & \mathrm{C} 3 \mathrm{~B}-\mathrm{C} 4 \mathrm{~B} & 1.313(3) \\ \mathrm{C} 1 \mathrm{~A}-\mathrm{C} 2 \mathrm{~A} & 1.5624(19) & \mathrm{C} 4 \mathrm{~B}-\mathrm{C} 5 \mathrm{~B} & 1.495(2) \\ \mathrm{C} 2 \mathrm{~A}-\mathrm{C} 3 \mathrm{~A} & 1.494(2) & \mathrm{C} 6 \mathrm{~B}-\mathrm{C} 7 \mathrm{~B} & 1.552(3) \\ \mathrm{C} 3 \mathrm{~A}-\mathrm{C} 4 \mathrm{~A} & 1.314(2) & \mathrm{C} 6 \mathrm{~B}-\mathrm{C} 10 \mathrm{~B} & 1.575(3) \\ \mathrm{C} 4 \mathrm{~A}-\mathrm{C} 5 \mathrm{~A} & 1.496(2) & \mathrm{C} 7 \mathrm{~B}-\mathrm{C} 8 \mathrm{~B} & 1.490(3) \\ \mathrm{C} 6 \mathrm{~A}-\mathrm{C} 7 \mathrm{~A} & 1.5453(18) & \mathrm{C} 8 \mathrm{~B}-\mathrm{C} 9 \mathrm{~B} & 1.313(3) \\ \mathrm{C} 6 \mathrm{~A}-\mathrm{C} 10 \mathrm{~A} & 1.5472(19) & \mathrm{C} 9 \mathrm{~B}-\mathrm{C} 10 \mathrm{~B} & 1.499(3) \\ \mathrm{C} 7 \mathrm{~A}-\mathrm{C} 8 \mathrm{~A} & 1.500(2) & \mathrm{O} 1 \mathrm{~B}-\mathrm{H} 1 \mathrm{~B} & 0.87(3) \\ \mathrm{C} 8 \mathrm{~A}-\mathrm{C} 9 \mathrm{~A} & 1.324(2) & \mathrm{O} 1 \mathrm{~B}-\mathrm{H} 1 \mathrm{C} & 0.87(3) \\ \mathrm{C} 9 \mathrm{~A}-\mathrm{C} 10 \mathrm{~A} & 1.490(2) & \mathrm{O} 1 \mathrm{C}-\mathrm{C} 1 \mathrm{C} & 1.418(9) \\ \mathrm{O} 1 \mathrm{~A}-\mathrm{H} 1 \mathrm{~A} & 0.90(3) & \mathrm{C} 1 \mathrm{C}-\mathrm{C} 6 \mathrm{C} & 1.543(7) \\ \text { O1B-C6B } & 1.419(4) & \mathrm{C} 6 \mathrm{C}-\mathrm{C} 11 \mathrm{C} & 1.492(7) \\ \mathrm{N} 1 \mathrm{~B}-\mathrm{C} 11 \mathrm{~B} & 1.180(4) & \mathrm{O} 1 \mathrm{C}-\mathrm{H} 1 \mathrm{~B} & 0.95(3) \\ \mathrm{C} 1 \mathrm{~B}-\mathrm{C} 11 \mathrm{~B} & 1.476(6) & \mathrm{O} 1 \mathrm{C}-\mathrm{H} 1 \mathrm{C} & 0.95(3)\end{array}$




\begin{tabular}{|c|c|c|c|}
\hline $\mathrm{C} 5 \mathrm{~A}-\mathrm{C} 1 \mathrm{~A}-\mathrm{C} 2 \mathrm{~A}$ & $105.72(11)$ & $\mathrm{C} 11 \mathrm{~B}-\mathrm{C} 1 \mathrm{~B}-\mathrm{C} 2 \mathrm{~B}$ & $111.1(2)$ \\
\hline $\mathrm{C} 5 \mathrm{~A}-\mathrm{C} 1 \mathrm{~A}-\mathrm{C} 6 \mathrm{~A}$ & $111.77(12)$ & $\mathrm{C} 11 \mathrm{~B}-\mathrm{C} 1 \mathrm{~B}-\mathrm{C} 5 \mathrm{~B}$ & $110.5(2)$ \\
\hline $\mathrm{C} 6 \mathrm{~A}-\mathrm{C} 1 \mathrm{~A}-\mathrm{C} 2 \mathrm{~A}$ & $112.28(11)$ & $\mathrm{C} 11 \mathrm{~B}-\mathrm{C} 1 \mathrm{~B}-\mathrm{C} 6 \mathrm{~B}$ & $108.9(3)$ \\
\hline $\mathrm{C} 11 \mathrm{~A}-\mathrm{C} 1 \mathrm{~A}-\mathrm{C} 2 \mathrm{~A}$ & $110.05(12)$ & $\mathrm{C} 3 \mathrm{~B}-\mathrm{C} 2 \mathrm{~B}-\mathrm{C} 1 \mathrm{~B}$ & $103.77(15)$ \\
\hline $\mathrm{C} 11 \mathrm{~A}-\mathrm{C} 1 \mathrm{~A}-\mathrm{C} 5 \mathrm{~A}$ & $109.36(11)$ & $\mathrm{C} 4 \mathrm{~B}-\mathrm{C} 3 \mathrm{~B}-\mathrm{C} 2 \mathrm{~B}$ & $112.39(15)$ \\
\hline $\mathrm{C} 11 \mathrm{~A}-\mathrm{C} 1 \mathrm{~A}-\mathrm{C} 6 \mathrm{~A}$ & $107.66(11)$ & $\mathrm{C} 3 \mathrm{~B}-\mathrm{C} 4 \mathrm{~B}-\mathrm{C} 5 \mathrm{~B}$ & $112.70(15)$ \\
\hline $\mathrm{C} 3 \mathrm{~A}-\mathrm{C} 2 \mathrm{~A}-\mathrm{C} 1 \mathrm{~A}$ & $103.41(12)$ & $\mathrm{C} 4 \mathrm{~B}-\mathrm{C} 5 \mathrm{~B}-\mathrm{C} 1 \mathrm{~B}$ & $104.53(15)$ \\
\hline $\mathrm{C} 4 \mathrm{~A}-\mathrm{C} 3 \mathrm{~A}-\mathrm{C} 2 \mathrm{~A}$ & $113.25(14)$ & $\mathrm{C} 1 \mathrm{~B}-\mathrm{C} 6 \mathrm{~B}-\mathrm{C} 7 \mathrm{~B}$ & $109.7(2)$ \\
\hline $\mathrm{C} 3 \mathrm{~A}-\mathrm{C} 4 \mathrm{~A}-\mathrm{C} 5 \mathrm{~A}$ & $112.82(13)$ & $\mathrm{C} 1 \mathrm{~B}-\mathrm{C} 6 \mathrm{~B}-\mathrm{C} 10 \mathrm{~B}$ & $110.9(2)$ \\
\hline $\mathrm{C} 4 \mathrm{~A}-\mathrm{C} 5 \mathrm{~A}-\mathrm{C} 1 \mathrm{~A}$ & $103.72(12)$ & $\mathrm{C} 7 \mathrm{~B}-\mathrm{C} 6 \mathrm{~B}-\mathrm{C} 10 \mathrm{~B}$ & $103.82(18)$ \\
\hline $\mathrm{C} 7 \mathrm{~A}-\mathrm{C} 6 \mathrm{~A}-\mathrm{C} 1 \mathrm{~A}$ & $112.63(11)$ & $\mathrm{O} 1 \mathrm{~B}-\mathrm{C} 6 \mathrm{~B}-\mathrm{C} 1 \mathrm{~B}$ & $108.7(3)$ \\
\hline $\mathrm{C} 7 \mathrm{~A}-\mathrm{C} 6 \mathrm{~A}-\mathrm{C} 10 \mathrm{~A}$ & $105.71(11)$ & $\mathrm{O} 1 \mathrm{~B}-\mathrm{C} 6 \mathrm{~B}-\mathrm{C} 7 \mathrm{~B}$ & $109.3(2)$ \\
\hline $\mathrm{C} 10 \mathrm{~A}-\mathrm{C} 6 \mathrm{~A}-\mathrm{C} 1 \mathrm{~A}$ & $112.38(11)$ & $\mathrm{O} 1 \mathrm{~B}-\mathrm{C} 6 \mathrm{~B}-\mathrm{C} 10 \mathrm{~B}$ & $114.2(2)$ \\
\hline $\mathrm{O} 1 \mathrm{~A}-\mathrm{C} 6 \mathrm{~A}-\mathrm{C} 1 \mathrm{~A}$ & $109.83(11)$ & $\mathrm{C} 8 \mathrm{~B}-\mathrm{C} 7 \mathrm{~B}-\mathrm{C} 6 \mathrm{~B}$ & $103.69(17)$ \\
\hline $\mathrm{O} 1 \mathrm{~A}-\mathrm{C} 6 \mathrm{~A}-\mathrm{C} 7 \mathrm{~A}$ & $106.07(11)$ & $\mathrm{C} 9 \mathrm{~B}-\mathrm{C} 8 \mathrm{~B}-\mathrm{C} 7 \mathrm{~B}$ & $112.06(16)$ \\
\hline $\mathrm{O} 1 \mathrm{~A}-\mathrm{C} 6 \mathrm{~A}-\mathrm{C} 10 \mathrm{~A}$ & $109.96(11)$ & $\mathrm{C} 8 \mathrm{~B}-\mathrm{C} 9 \mathrm{~B}-\mathrm{C} 10 \mathrm{~B}$ & $113.14(17)$ \\
\hline $\mathrm{C} 8 \mathrm{~A}-\mathrm{C} 7 \mathrm{~A}-\mathrm{C} 6 \mathrm{~A}$ & $103.67(12)$ & $\mathrm{C} 9 \mathrm{~B}-\mathrm{C} 10 \mathrm{~B}-\mathrm{C} 6 \mathrm{~B}$ & $102.19(16)$ \\
\hline $\mathrm{C} 9 \mathrm{~A}-\mathrm{C} 8 \mathrm{~A}-\mathrm{C} 7 \mathrm{~A}$ & $112.44(14)$ & $\mathrm{N} 1 \mathrm{~B}-\mathrm{C} 11 \mathrm{~B}-\mathrm{C} 1 \mathrm{~B}$ & $176.9(4)$ \\
\hline $\mathrm{C} 8 \mathrm{~A}-\mathrm{C} 9 \mathrm{~A}-\mathrm{C} 10 \mathrm{~A}$ & $112.44(14)$ & $\mathrm{C} 6 \mathrm{~B}-\mathrm{O} 1 \mathrm{~B}-\mathrm{H} 1 \mathrm{~B}$ & $113.3(19)$ \\
\hline $\mathrm{C} 9 \mathrm{~A}-\mathrm{C} 10 \mathrm{~A}-\mathrm{C} 6 \mathrm{~A}$ & $104.05(11)$ & $\mathrm{C} 6 \mathrm{~B}-\mathrm{O} 1 \mathrm{~B}-\mathrm{H} 1 \mathrm{C}$ & $113.3(19)$ \\
\hline $\mathrm{N} 1 \mathrm{~A}-\mathrm{C} 11 \mathrm{~A}-\mathrm{C} 1 \mathrm{~A}$ & $178.51(15)$ & $\mathrm{O} 1 \mathrm{C}-\mathrm{C} 1 \mathrm{C}-\mathrm{C} 6 \mathrm{C}$ & $108.0(5)$ \\
\hline $\mathrm{C} 6 \mathrm{~A}-\mathrm{O} 1 \mathrm{~A}-\mathrm{H} 1 \mathrm{~A}$ & $113.3(16)$ & $\mathrm{C} 11 \mathrm{C}-\mathrm{C} 6 \mathrm{C}-\mathrm{C} 1 \mathrm{C}$ & $109.0(5)$ \\
\hline $\mathrm{C} 5 \mathrm{~B}-\mathrm{C} 1 \mathrm{~B}-\mathrm{C} 2 \mathrm{~B}$ & $103.36(19)$ & $\mathrm{C} 1 \mathrm{C}-\mathrm{O} 1 \mathrm{C}-\mathrm{H} 1 \mathrm{C}$ & $115.9(18)$ \\
\hline $\mathrm{C} 6 \mathrm{~B}-\mathrm{C} 1 \mathrm{~B}-\mathrm{C} 2 \mathrm{~B}$ & $111.5(3)$ & $\mathrm{C} 1 \mathrm{C}-\mathrm{O} 1 \mathrm{C}-\mathrm{H} 1 \mathrm{~B}$ & $115.9(18)$ \\
\hline $\mathrm{C} 6 \mathrm{~B}-\mathrm{C} 1 \mathrm{~B}-\mathrm{C} 5 \mathrm{~B}$ & $111.4(3)$ & & \\
\hline $\mathrm{C} 11 \mathrm{~A}-\mathrm{C} 1 \mathrm{~A}-\mathrm{C} 2 \mathrm{~A}-\mathrm{C} 3 \mathrm{~A}$ & $127.62(13)$ & $\mathrm{C} 6 \mathrm{~B}-\mathrm{C} 1 \mathrm{~B}-\mathrm{C} 2 \mathrm{~B}-\mathrm{C} 3 \mathrm{~B}$ & $-136.6(2)$ \\
\hline $\mathrm{C} 5 \mathrm{~A}-\mathrm{C} 1 \mathrm{~A}-\mathrm{C} 2 \mathrm{~A}-\mathrm{C} 3 \mathrm{~A}$ & $9.63(15)$ & $\mathrm{C} 5 \mathrm{~B}-\mathrm{C} 1 \mathrm{~B}-\mathrm{C} 2 \mathrm{~B}-\mathrm{C} 3 \mathrm{~B}$ & $-16.8(3)$ \\
\hline $\mathrm{C} 6 \mathrm{~A}-\mathrm{C} 1 \mathrm{~A}-\mathrm{C} 2 \mathrm{~A}-\mathrm{C} 3 \mathrm{~A}$ & $-112.49(13)$ & $\mathrm{C} 1 \mathrm{~B}-\mathrm{C} 2 \mathrm{~B}-\mathrm{C} 3 \mathrm{~B}-\mathrm{C} 4 \mathrm{~B}$ & $11.1(2)$ \\
\hline $\mathrm{C} 1 \mathrm{~A}-\mathrm{C} 2 \mathrm{~A}-\mathrm{C} 3 \mathrm{~A}-\mathrm{C} 4 \mathrm{~A}$ & $-5.79(18)$ & $\mathrm{C} 2 \mathrm{~B}-\mathrm{C} 3 \mathrm{~B}-\mathrm{C} 4 \mathrm{~B}-\mathrm{C} 5 \mathrm{~B}$ & $0.1(2)$ \\
\hline $\mathrm{C} 2 \mathrm{~A}-\mathrm{C} 3 \mathrm{~A}-\mathrm{C} 4 \mathrm{~A}-\mathrm{C} 5 \mathrm{~A}$ & $-0.8(2)$ & $\mathrm{C} 3 \mathrm{~B}-\mathrm{C} 4 \mathrm{~B}-\mathrm{C} 5 \mathrm{~B}-\mathrm{C} 1 \mathrm{~B}$ & $-11.5(2)$ \\
\hline $\mathrm{C} 3 \mathrm{~A}-\mathrm{C} 4 \mathrm{~A}-\mathrm{C} 5 \mathrm{~A}-\mathrm{C} 1 \mathrm{~A}$ & $7.08(18)$ & $\mathrm{C} 11 \mathrm{~B}-\mathrm{C} 1 \mathrm{~B}-\mathrm{C} 5 \mathrm{~B}-\mathrm{C} 4 \mathrm{~B}$ & $-102.0(2)$ \\
\hline $\mathrm{C} 11 \mathrm{~A}-\mathrm{C} 1 \mathrm{~A}-\mathrm{C} 5 \mathrm{~A}-\mathrm{C} 4 \mathrm{~A}$ & $-128.51(13)$ & $\mathrm{C} 6 \mathrm{~B}-\mathrm{C} 1 \mathrm{~B}-\mathrm{C} 5 \mathrm{~B}-\mathrm{C} 4 \mathrm{~B}$ & $136.8(2)$ \\
\hline $\mathrm{C} 6 \mathrm{~A}-\mathrm{C} 1 \mathrm{~A}-\mathrm{C} 5 \mathrm{~A}-\mathrm{C} 4 \mathrm{~A}$ & $112.38(13)$ & $\mathrm{C} 2 \mathrm{~B}-\mathrm{C} 1 \mathrm{~B}-\mathrm{C} 5 \mathrm{~B}-\mathrm{C} 4 \mathrm{~B}$ & $17.0(3)$ \\
\hline $\mathrm{C} 2 \mathrm{~A}-\mathrm{C} 1 \mathrm{~A}-\mathrm{C} 5 \mathrm{~A}-\mathrm{C} 4 \mathrm{~A}$ & $-10.06(15)$ & $\mathrm{C} 11 \mathrm{~B}-\mathrm{C} 1 \mathrm{~B}-\mathrm{C} 6 \mathrm{~B}-\mathrm{O} 1 \mathrm{~B}$ & $-177.0(2)$ \\
\hline
\end{tabular}




\begin{tabular}{|c|c|c|c|}
\hline $\mathrm{C} 11 \mathrm{~A}-\mathrm{C} 1 \mathrm{~A}-\mathrm{C} 6 \mathrm{~A}-\mathrm{O} 1 \mathrm{~A}$ & $67.25(14)$ & $\mathrm{C} 5 \mathrm{~B}-\mathrm{C} 1 \mathrm{~B}-\mathrm{C} 6 \mathrm{~B}-\mathrm{O} 1 \mathrm{~B}$ & $-54.9(3)$ \\
\hline $\mathrm{C} 5 \mathrm{~A}-\mathrm{C} 1 \mathrm{~A}-\mathrm{C} 6 \mathrm{~A}-\mathrm{O} 1 \mathrm{~A}$ & $-172.64(11)$ & $\mathrm{C} 2 \mathrm{~B}-\mathrm{C} 1 \mathrm{~B}-\mathrm{C} 6 \mathrm{~B}-\mathrm{O} 1 \mathrm{~B}$ & $60.0(3)$ \\
\hline $\mathrm{C} 2 \mathrm{~A}-\mathrm{C} 1 \mathrm{~A}-\mathrm{C} 6 \mathrm{~A}-\mathrm{O} 1 \mathrm{~A}$ & $-54.03(14)$ & $\mathrm{C} 11 \mathrm{~B}-\mathrm{C} 1 \mathrm{~B}-\mathrm{C} 6 \mathrm{~B}-\mathrm{C} 7 \mathrm{~B}$ & $-57.5(3)$ \\
\hline $\mathrm{C} 11 \mathrm{~A}-\mathrm{C} 1 \mathrm{~A}-\mathrm{C} 6 \mathrm{~A}-\mathrm{C} 7 \mathrm{~A}$ & $-174.77(11)$ & $\mathrm{C} 5 \mathrm{~B}-\mathrm{C} 1 \mathrm{~B}-\mathrm{C} 6 \mathrm{~B}-\mathrm{C} 7 \mathrm{~B}$ & $64.6(4)$ \\
\hline $\mathrm{C} 5 \mathrm{~A}-\mathrm{C} 1 \mathrm{~A}-\mathrm{C} 6 \mathrm{~A}-\mathrm{C} 7 \mathrm{~A}$ & $-54.66(15)$ & $\mathrm{C} 2 \mathrm{~B}-\mathrm{C} 1 \mathrm{~B}-\mathrm{C} 6 \mathrm{~B}-\mathrm{C} 7 \mathrm{~B}$ & $179.54(19)$ \\
\hline $\mathrm{C} 2 \mathrm{~A}-\mathrm{C} 1 \mathrm{~A}-\mathrm{C} 6 \mathrm{~A}-\mathrm{C} 7 \mathrm{~A}$ & $63.95(15)$ & $\mathrm{C} 11 \mathrm{~B}-\mathrm{C} 1 \mathrm{~B}-\mathrm{C} 6 \mathrm{~B}-\mathrm{C} 10 \mathrm{~B}$ & $56.6(3)$ \\
\hline $\mathrm{C} 11 \mathrm{~A}-\mathrm{C} 1 \mathrm{~A}-\mathrm{C} 6 \mathrm{~A}-\mathrm{C} 10 \mathrm{~A}$ & $-55.50(15)$ & $\mathrm{C} 5 \mathrm{~B}-\mathrm{C} 1 \mathrm{~B}-\mathrm{C} 6 \mathrm{~B}-\mathrm{C} 10 \mathrm{~B}$ & $178.76(19)$ \\
\hline $\mathrm{C} 5 \mathrm{~A}-\mathrm{C} 1 \mathrm{~A}-\mathrm{C} 6 \mathrm{~A}-\mathrm{C} 10 \mathrm{~A}$ & $64.61(14)$ & $\mathrm{C} 2 \mathrm{~B}-\mathrm{C} 1 \mathrm{~B}-\mathrm{C} 6 \mathrm{~B}-\mathrm{C} 10 \mathrm{~B}$ & $-66.3(4)$ \\
\hline $\mathrm{C} 2 \mathrm{~A}-\mathrm{C} 1 \mathrm{~A}-\mathrm{C} 6 \mathrm{~A}-\mathrm{C} 10 \mathrm{~A}$ & $-176.78(11)$ & $\mathrm{O} 1 \mathrm{~B}-\mathrm{C} 6 \mathrm{~B}-\mathrm{C} 7 \mathrm{~B}-\mathrm{C} 8 \mathrm{~B}$ & $-100.8(2)$ \\
\hline $\mathrm{O} 1 \mathrm{~A}-\mathrm{C} 6 \mathrm{~A}-\mathrm{C} 7 \mathrm{~A}-\mathrm{C} 8 \mathrm{~A}$ & $-104.46(13)$ & $\mathrm{C} 1 \mathrm{~B}-\mathrm{C} 6 \mathrm{~B}-\mathrm{C} 7 \mathrm{~B}-\mathrm{C} 8 \mathrm{~B}$ & $140.0(2)$ \\
\hline $\mathrm{C} 10 \mathrm{~A}-\mathrm{C} 6 \mathrm{~A}-\mathrm{C} 7 \mathrm{~A}-\mathrm{C} 8 \mathrm{~A}$ & $12.29(15)$ & $\mathrm{C} 10 \mathrm{~B}-\mathrm{C} 6 \mathrm{~B}-\mathrm{C} 7 \mathrm{~B}-\mathrm{C} 8 \mathrm{~B}$ & $21.4(3)$ \\
\hline $\mathrm{C} 1 \mathrm{~A}-\mathrm{C} 6 \mathrm{~A}-\mathrm{C} 7 \mathrm{~A}-\mathrm{C} 8 \mathrm{~A}$ & $135.37(12)$ & $\mathrm{C} 6 \mathrm{~B}-\mathrm{C} 7 \mathrm{~B}-\mathrm{C} 8 \mathrm{~B}-\mathrm{C} 9 \mathrm{~B}$ & $-14.4(3)$ \\
\hline $\mathrm{C} 6 \mathrm{~A}-\mathrm{C} 7 \mathrm{~A}-\mathrm{C} 8 \mathrm{~A}-\mathrm{C} 9 \mathrm{~A}$ & $-7.82(18)$ & $\mathrm{C} 7 \mathrm{~B}-\mathrm{C} 8 \mathrm{~B}-\mathrm{C} 9 \mathrm{~B}-\mathrm{C} 10 \mathrm{~B}$ & $0.4(2)$ \\
\hline $\mathrm{C} 7 \mathrm{~A}-\mathrm{C} 8 \mathrm{~A}-\mathrm{C} 9 \mathrm{~A}-\mathrm{C} 10 \mathrm{~A}$ & $-0.30(19)$ & $\mathrm{C} 8 \mathrm{~B}-\mathrm{C} 9 \mathrm{~B}-\mathrm{C} 10 \mathrm{~B}-\mathrm{C} 6 \mathrm{~B}$ & $13.5(2)$ \\
\hline $\mathrm{C} 8 \mathrm{~A}-\mathrm{C} 9 \mathrm{~A}-\mathrm{C} 10 \mathrm{~A}-\mathrm{C} 6 \mathrm{~A}$ & $8.30(17)$ & $\mathrm{O} 1 \mathrm{~B}-\mathrm{C} 6 \mathrm{~B}-\mathrm{C} 10 \mathrm{~B}-\mathrm{C} 9 \mathrm{~B}$ & $98.0(2)$ \\
\hline $\mathrm{O} 1 \mathrm{~A}-\mathrm{C} 6 \mathrm{~A}-\mathrm{C} 10 \mathrm{~A}-\mathrm{C} 9 \mathrm{~A}$ & $101.58(13)$ & $\mathrm{C} 1 \mathrm{~B}-\mathrm{C} 6 \mathrm{~B}-\mathrm{C} 10 \mathrm{~B}-\mathrm{C} 9 \mathrm{~B}$ & $-138.7(2)$ \\
\hline $\mathrm{C} 7 \mathrm{~A}-\mathrm{C} 6 \mathrm{~A}-\mathrm{C} 10 \mathrm{~A}-\mathrm{C} 9 \mathrm{~A}$ & $-12.50(14)$ & $\mathrm{C} 7 \mathrm{~B}-\mathrm{C} 6 \mathrm{~B}-\mathrm{C} 10 \mathrm{~B}-\mathrm{C} 9 \mathrm{~B}$ & $-20.9(2)$ \\
\hline $\mathrm{C} 1 \mathrm{~A}-\mathrm{C} 6 \mathrm{~A}-\mathrm{C} 10 \mathrm{~A}-\mathrm{C} 9 \mathrm{~A}$ & $-135.74(12)$ & $\mathrm{C} 11 \mathrm{~B}-\mathrm{C} 1 \mathrm{~B}-\mathrm{C} 2 \mathrm{~B}-\mathrm{C} 3 \mathrm{~B}$ & $101.6(2)$ \\
\hline $\mathrm{O} 1 \mathrm{C}-\mathrm{C} 1 \mathrm{C}-\mathrm{C} 6 \mathrm{C}-\mathrm{C} 11 \mathrm{C}$ & $176.9(3)$ & & \\
\hline
\end{tabular}


Figure S1 Molecule A Gauche Form

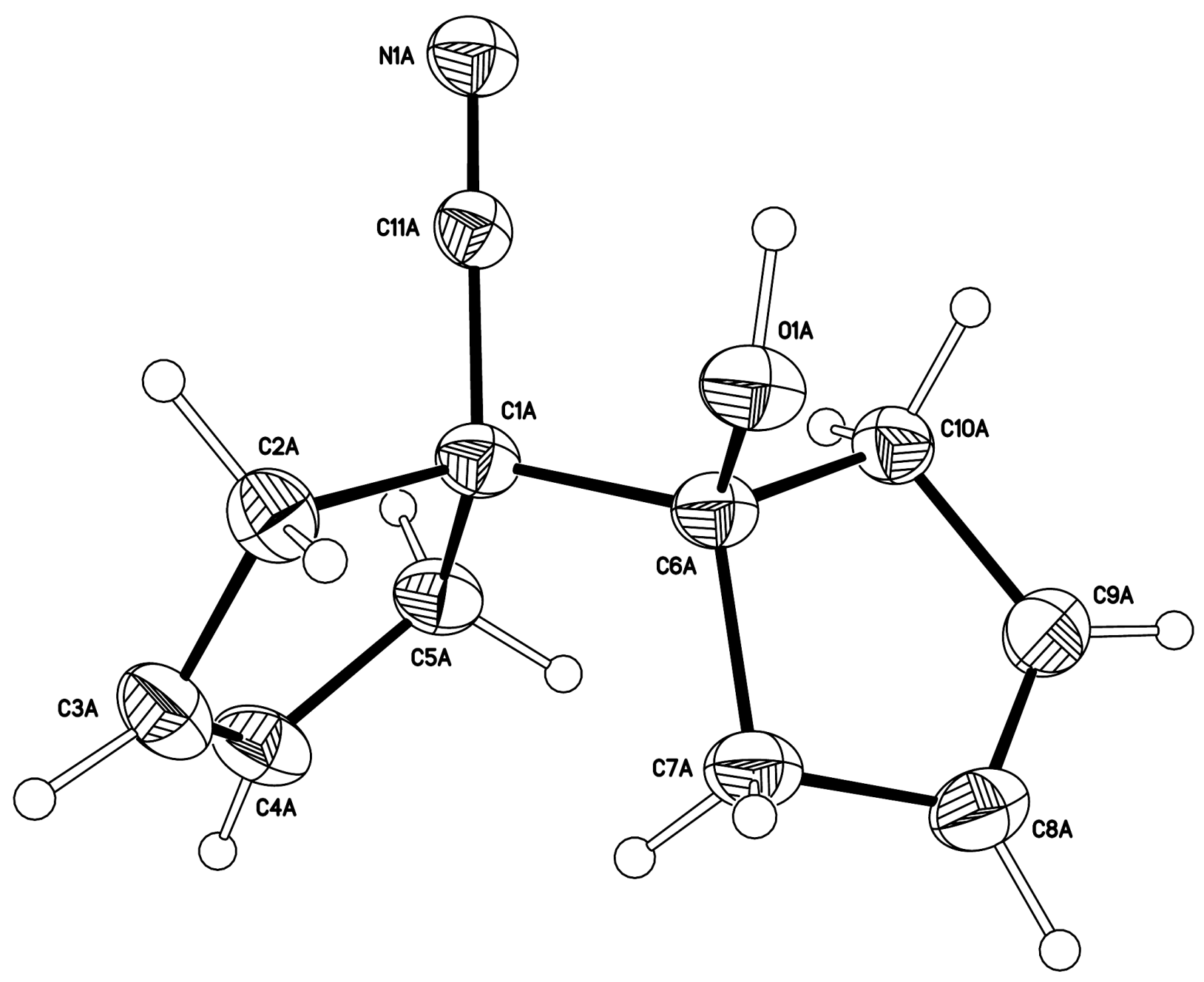


Figure S2 Molecule B and C Major and Minor components of the Anti Form
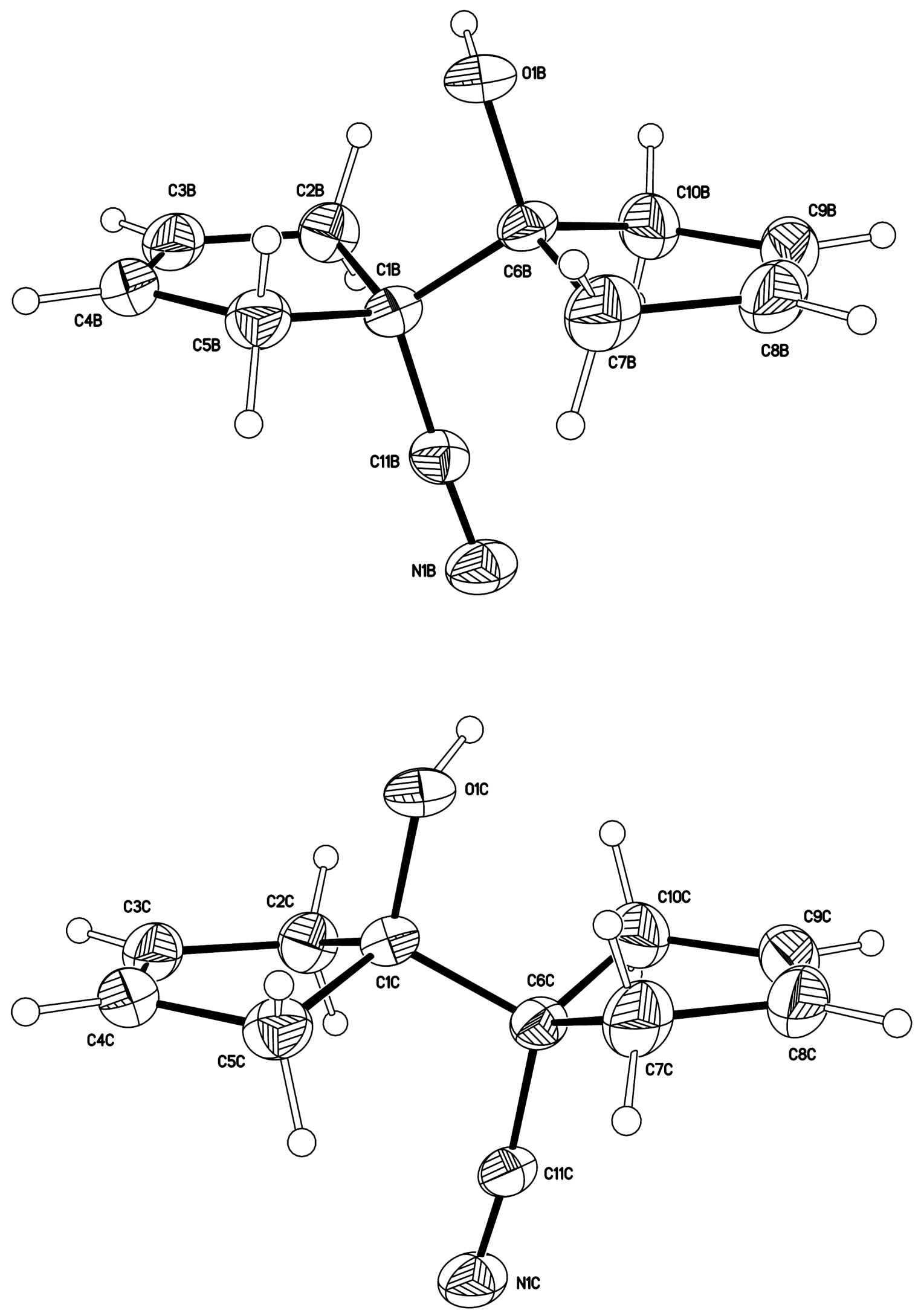
Figure S3 Molecules B and C Major and Minor components of the Anti Form showing disorder model

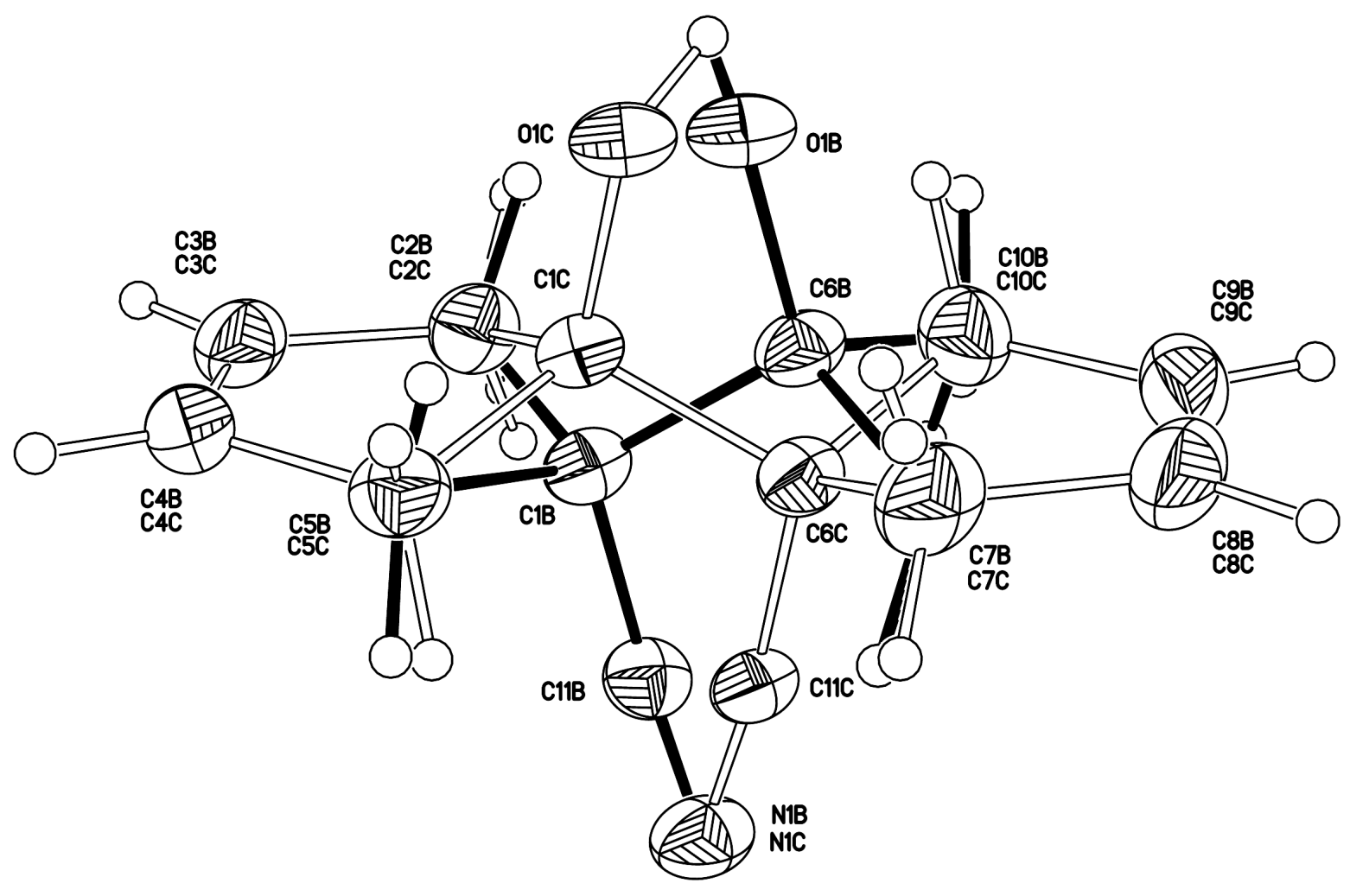


NMR spectra of all Compounds

${ }^{1} \mathrm{H}$ NMR ( $\left.\mathrm{CDCl}_{3}, 400 \mathrm{MHz}\right)$ Ethyl 2-allyl-2-cyanopent-4-enoate 12. ${ }^{5}$

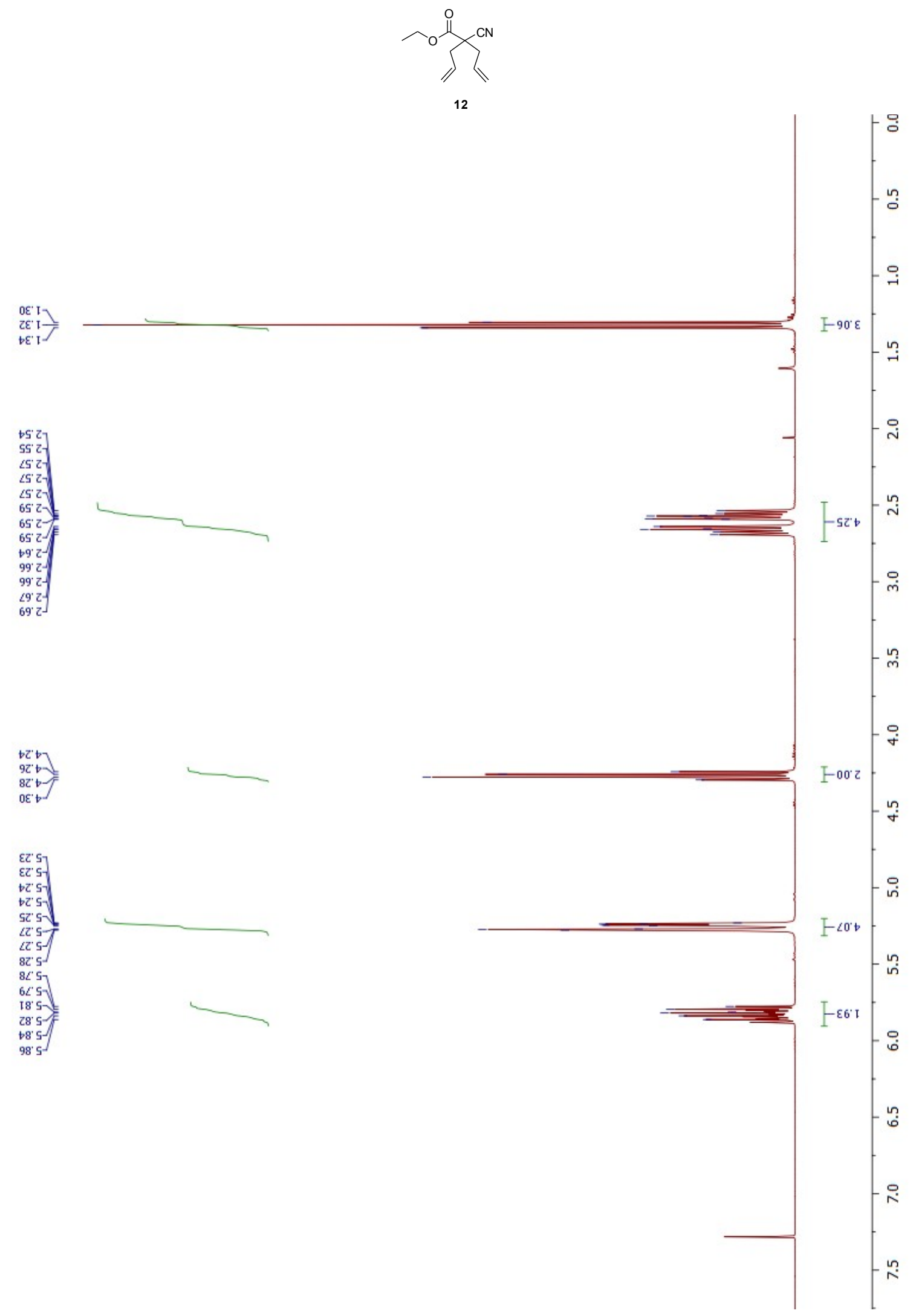


${ }^{13} \mathrm{C}$ NMR ( $\left.\mathrm{CDCl}_{3}, 100 \mathrm{MHz}\right)$ Ethyl 2-allyl-2-cyanopent-4-enoate 12.5

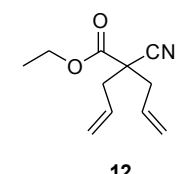

12

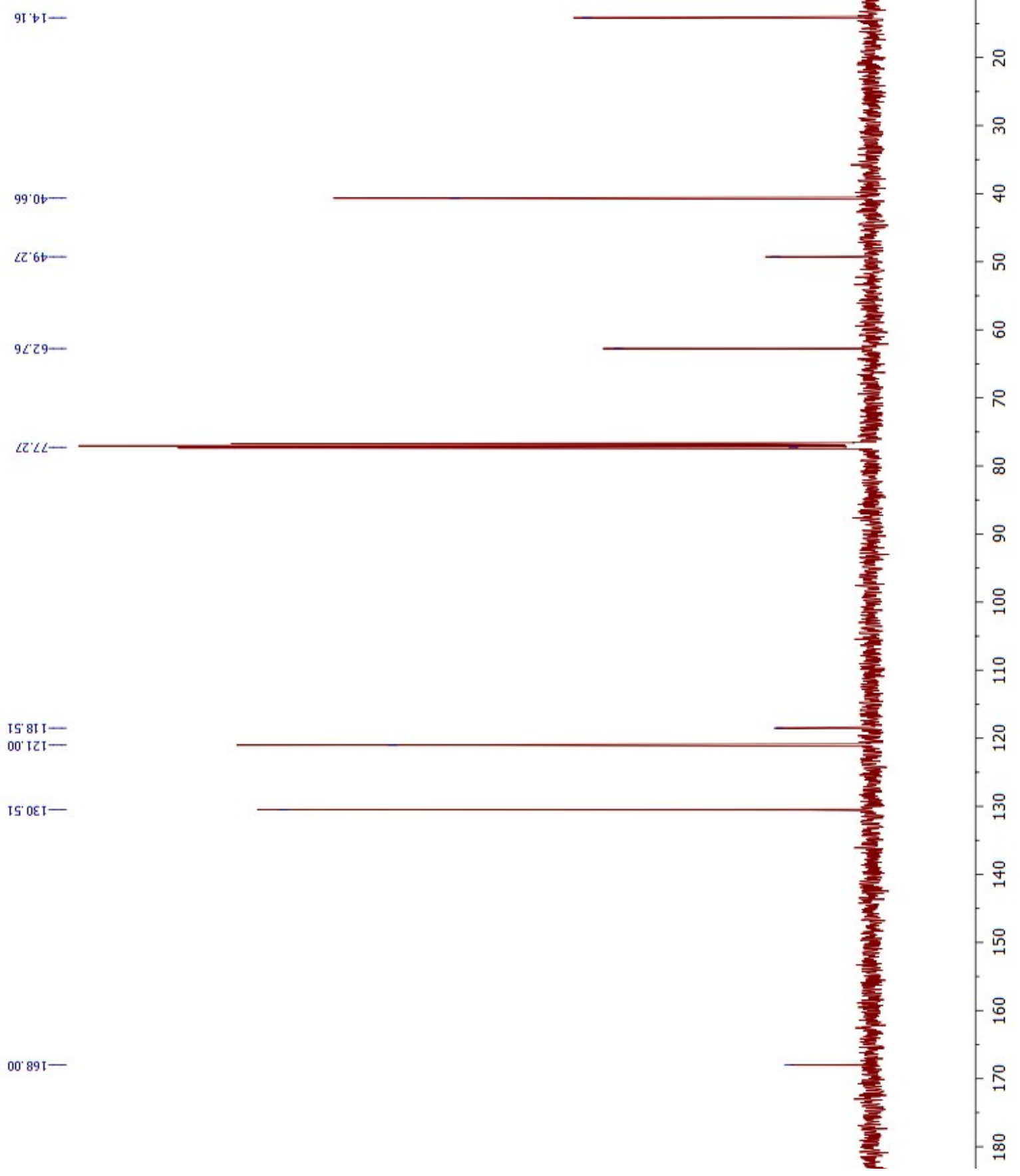


${ }^{1}$ H NMR (Methanol-d ${ }_{4}, 400$ MHz) 2-Allyl-2-cyanopent-4-enoic acid 13.

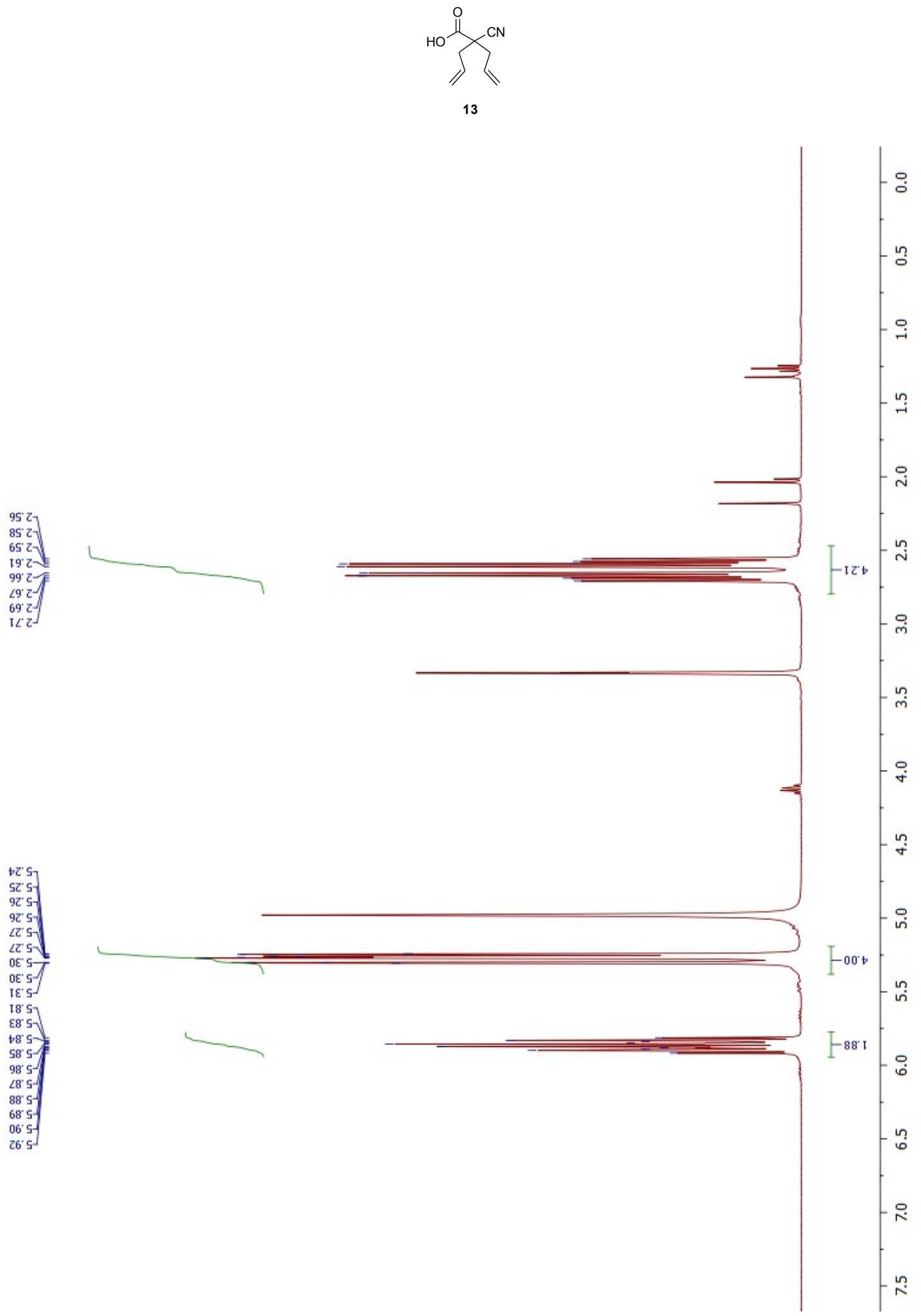


${ }^{13} \mathrm{C}$ NMR (Methanol-d $d_{4}, 100 \mathrm{MHz}$ ) 2-Allyl-2-cyanopent-4-enoic acid 13.

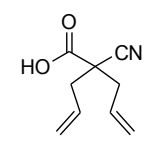

13

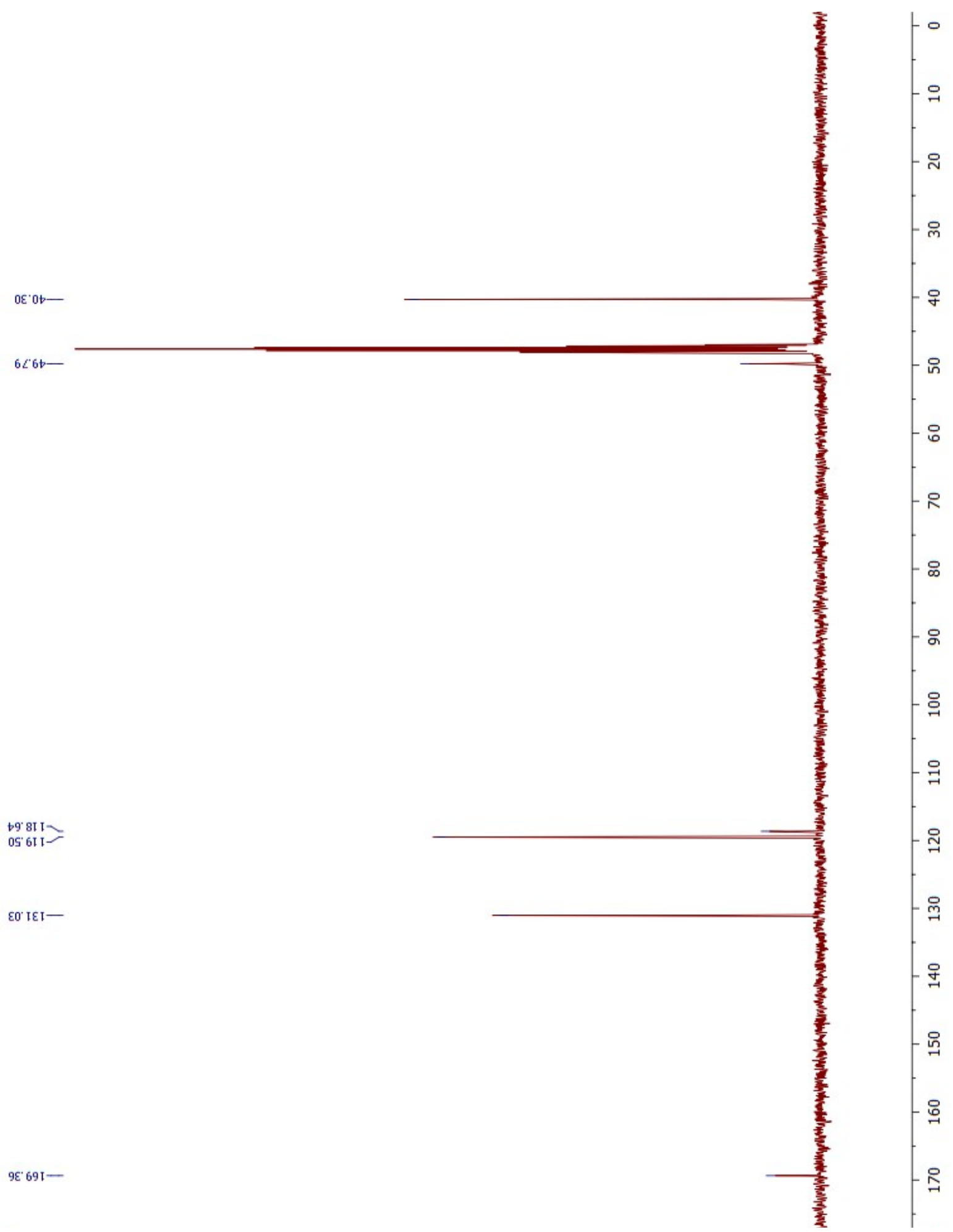


${ }^{1} \mathrm{H}$ NMR (CDCl $\left.\mathbf{3}, \mathbf{4 0 0} \mathbf{M H z}\right)$ 2,2,3-Triallyl-3-hydroxyhex-5-enenitrile 6.

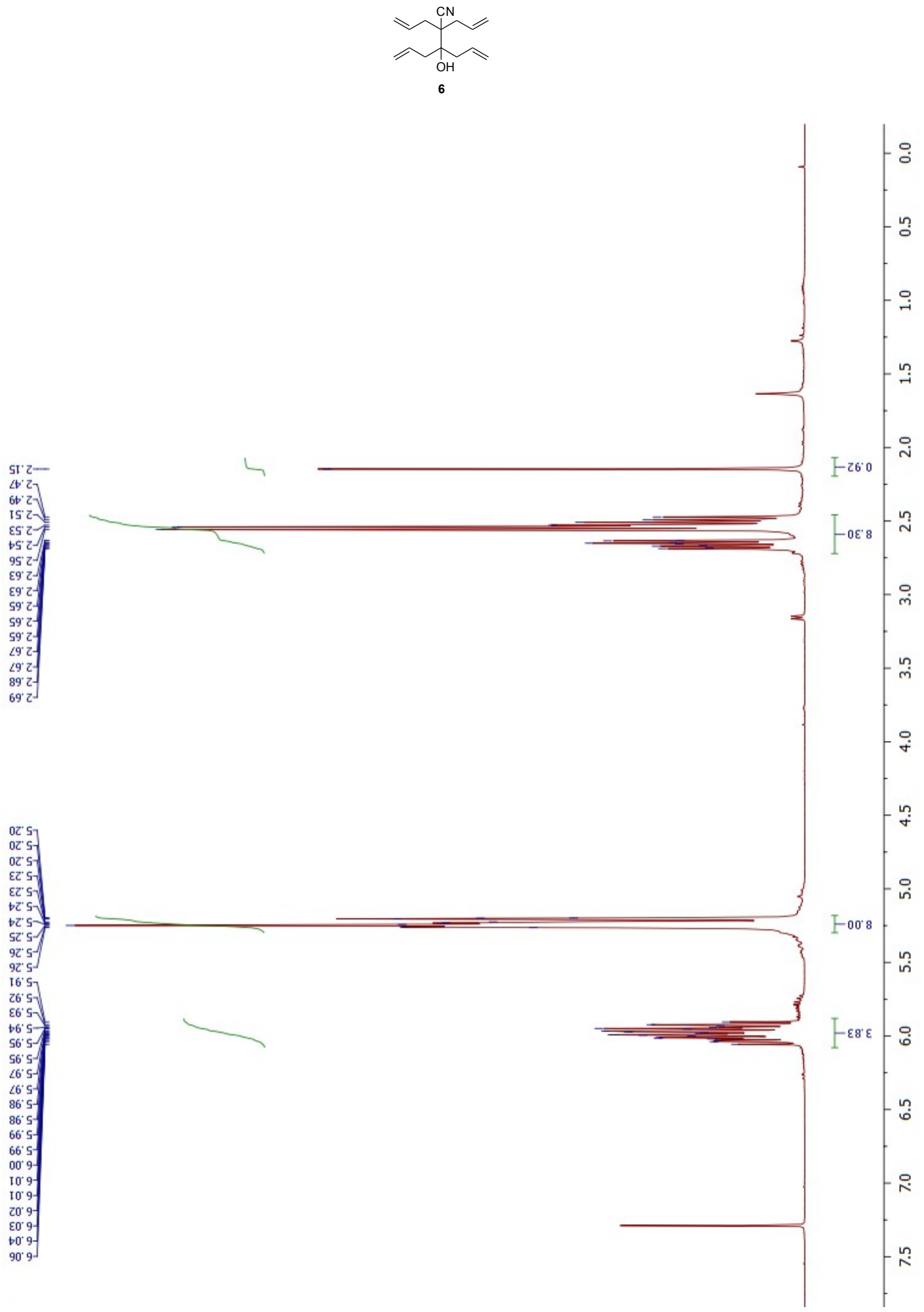


${ }^{13} \mathbf{C}$ NMR ( $\left.\mathbf{C D C l}_{\mathbf{3}}, \mathbf{1 0 0} \mathbf{M H z}\right) \quad$ 2,2,3-Triallyl-3-hydroxyhex-5-enenitrile 6.

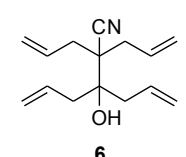

$6 \tau^{\prime} \angle \varepsilon-$

¿ย' It -

$0 b \cdot 0 s-$

$88^{\prime} 5<-$

$96^{\circ} 6 \mathrm{II}$

$86.6 \mathrm{II}$

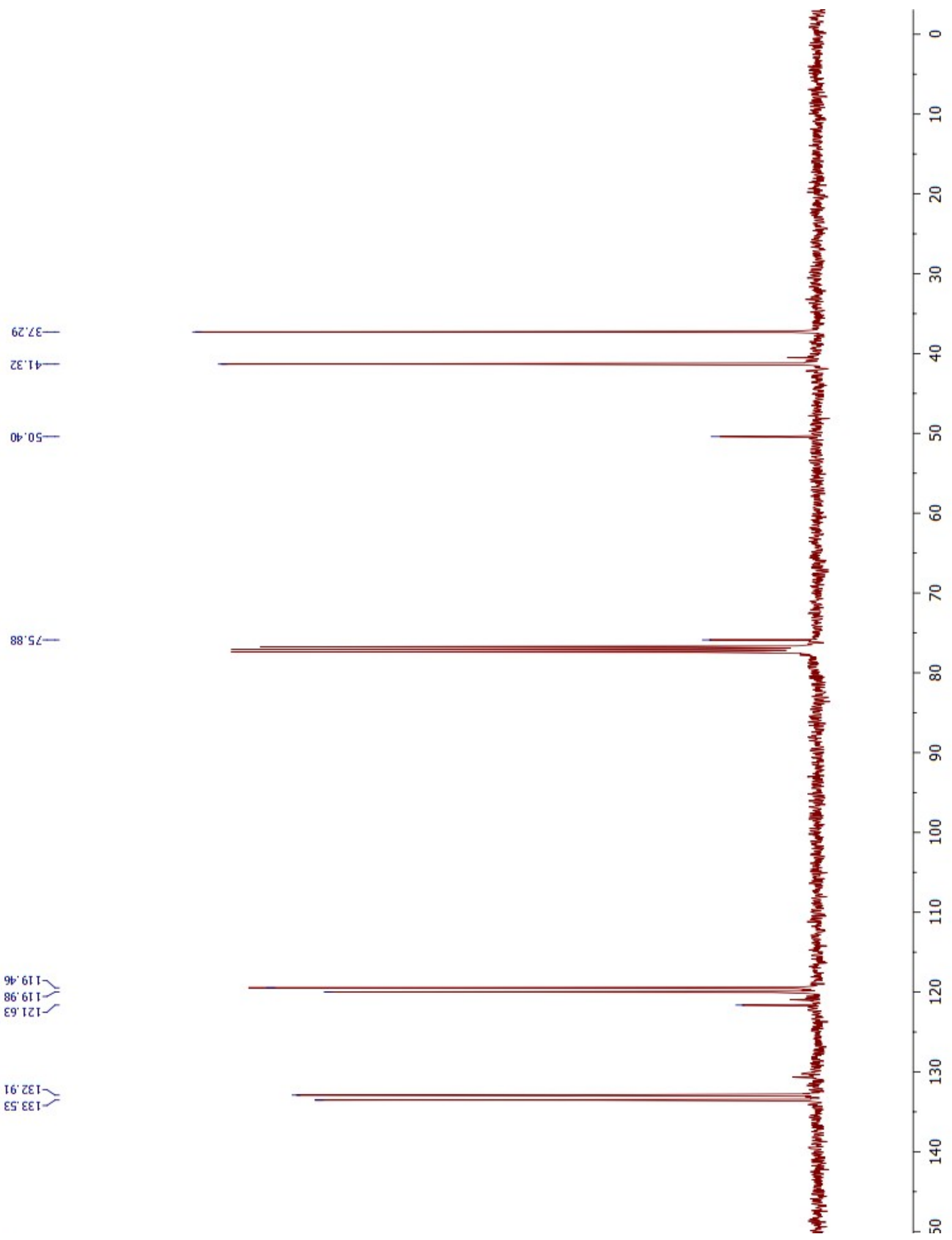


${ }^{1} \mathrm{H}$ NMR ( $\left.\mathrm{CDCl}_{3}, 400 \mathrm{MHz}\right)$ Ethyl 1-cyanocyclopent-3-ene-1-carboxylate 14. ${ }^{6}$

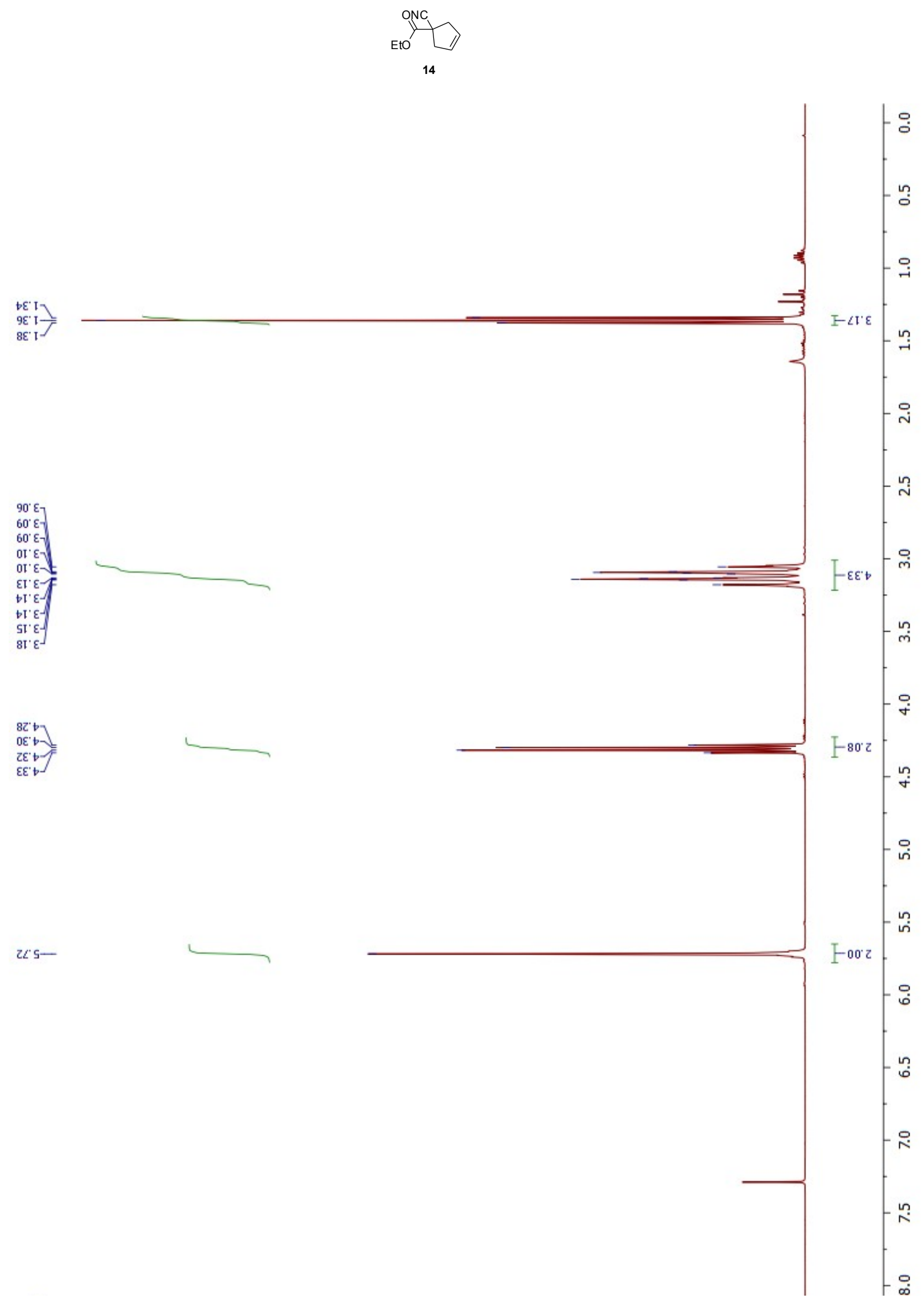


${ }^{13} \mathrm{C}$ NMR $\left(\mathrm{CDCl}_{3}, 100 \mathrm{MHz}\right)$ Ethyl 1-cyanocyclopent-3-ene-1-carboxylate 14. ${ }^{6}$

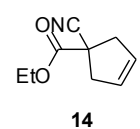

14

$66^{\cdot} \varepsilon \mathrm{I}-$

$80^{\circ} \varepsilon 9-$

oZ $\mathrm{IZI}$

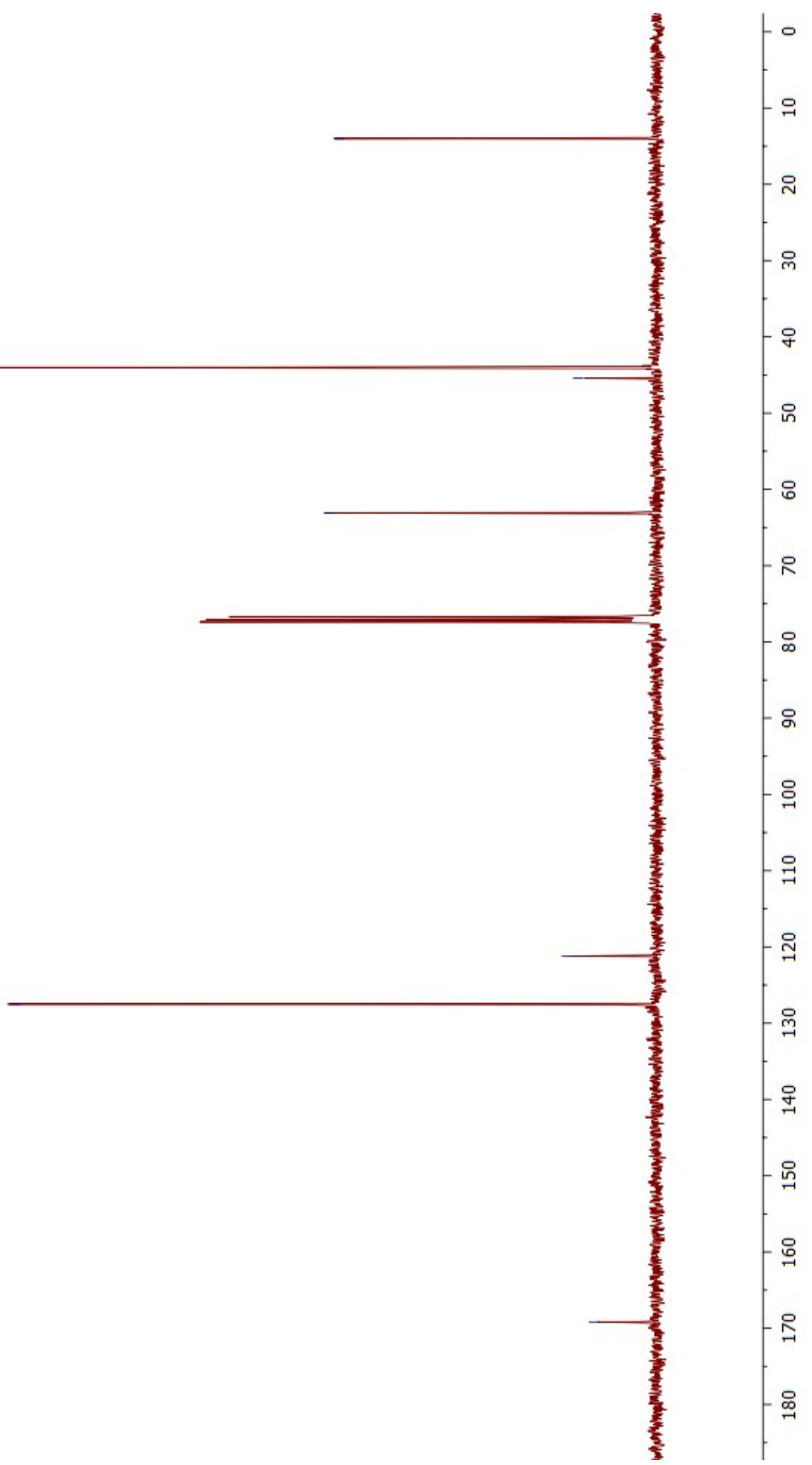


${ }^{1} \mathrm{H}$ NMR ( $\left.\mathrm{CDCl}_{3}, 400 \mathrm{MHz}\right)$ 1-Cyanocyclopent-3-ene-1-carboxylic acid 15.

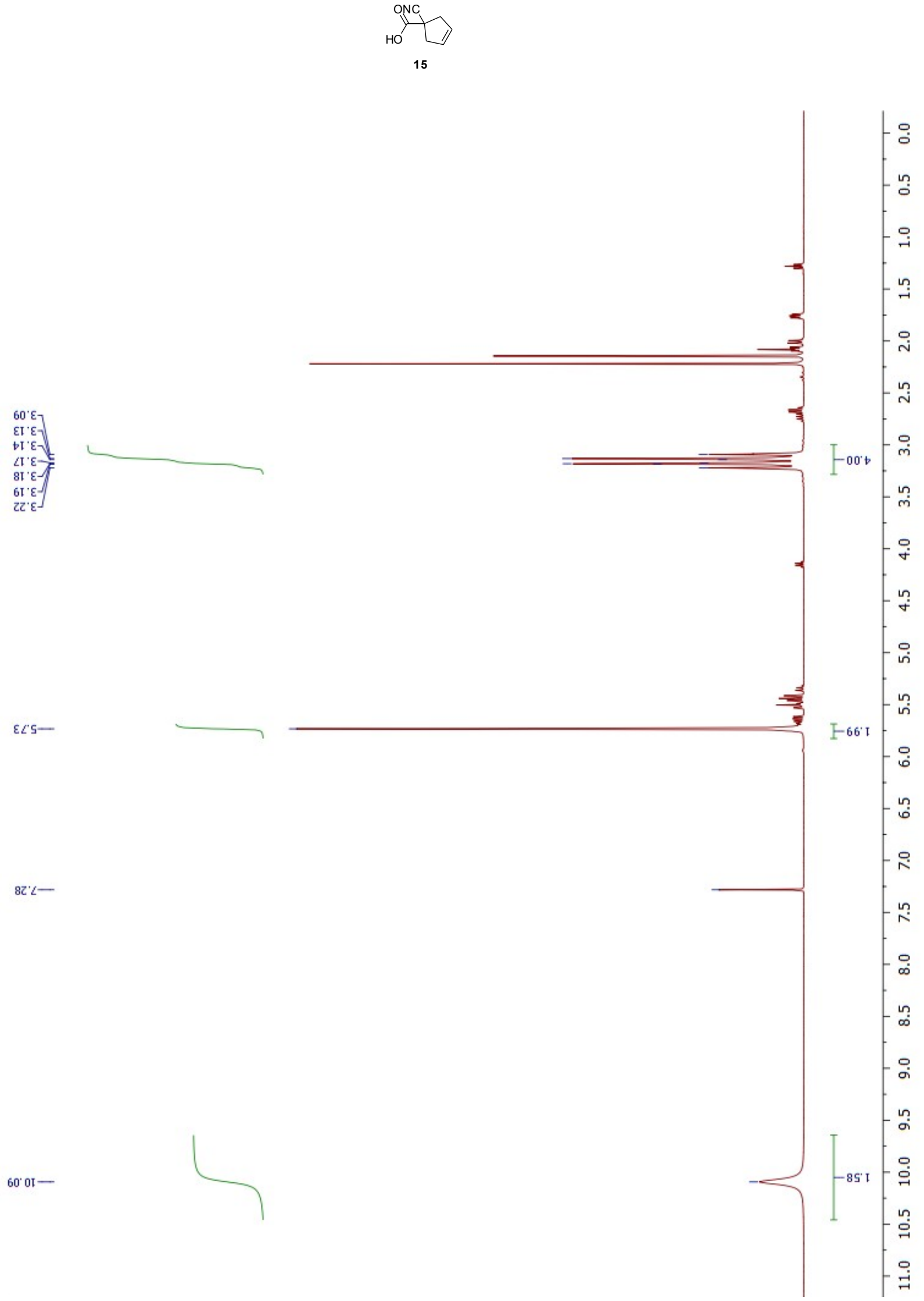


${ }^{13} \mathrm{C}$ NMR (Methanol- $d_{4}, 100 \mathrm{MHz}$ ) 1-Cyanocyclopent-3-ene-1-carboxylic acid 15.

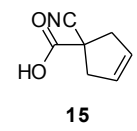

$\downarrow 6^{\circ} \varepsilon b-$

$90 \angle t$

90' દZI-

ob $\angle Z I-$

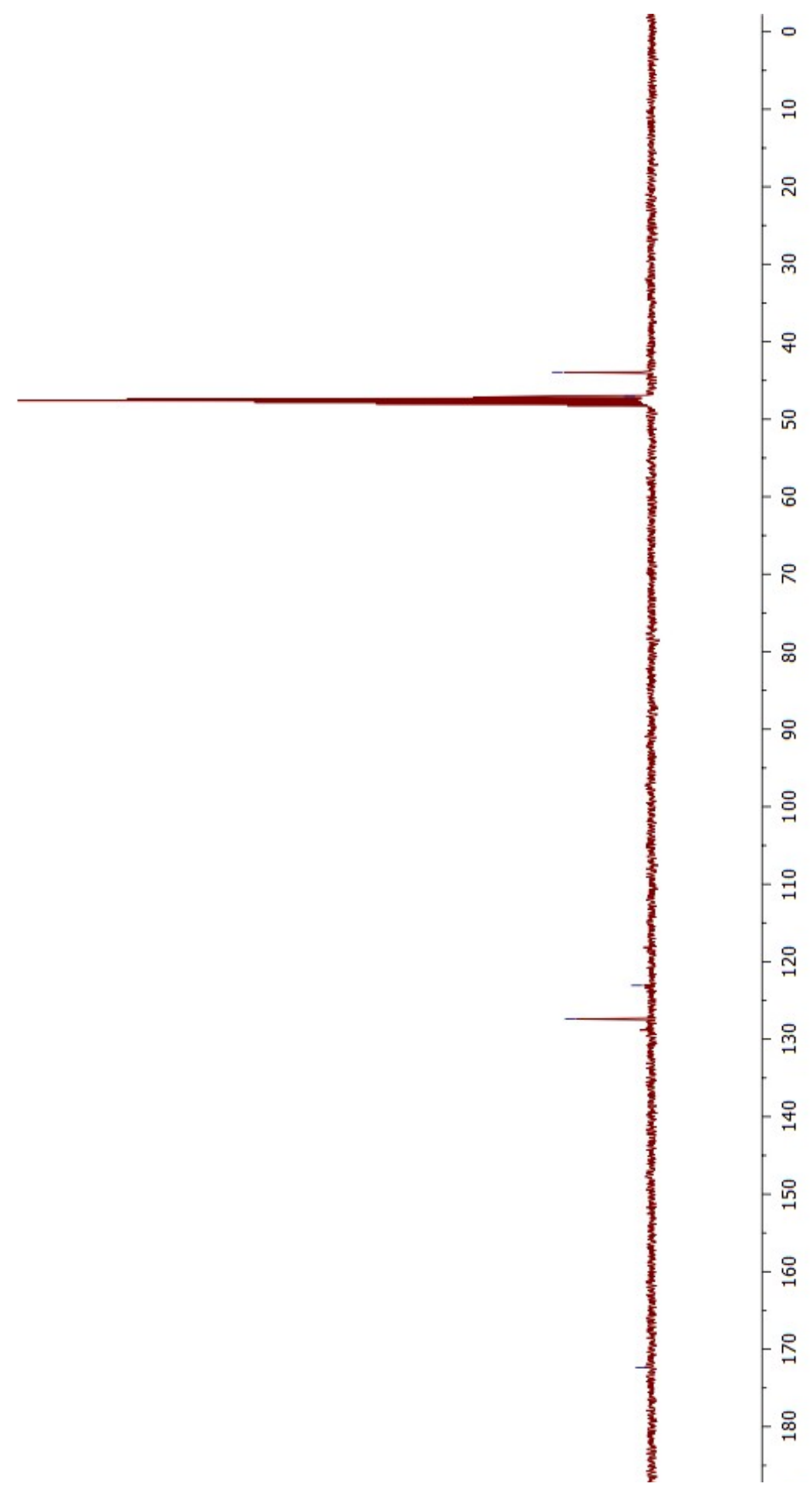


${ }^{1} \mathrm{H}$ NMR $\left(\mathbf{C D C l}_{3}, \quad 400\right.$ MHz) 1-(4-Hydroxyhepta-1,6-dien-4-yl)cyclopent-3-ene-1carbonitrile 7.

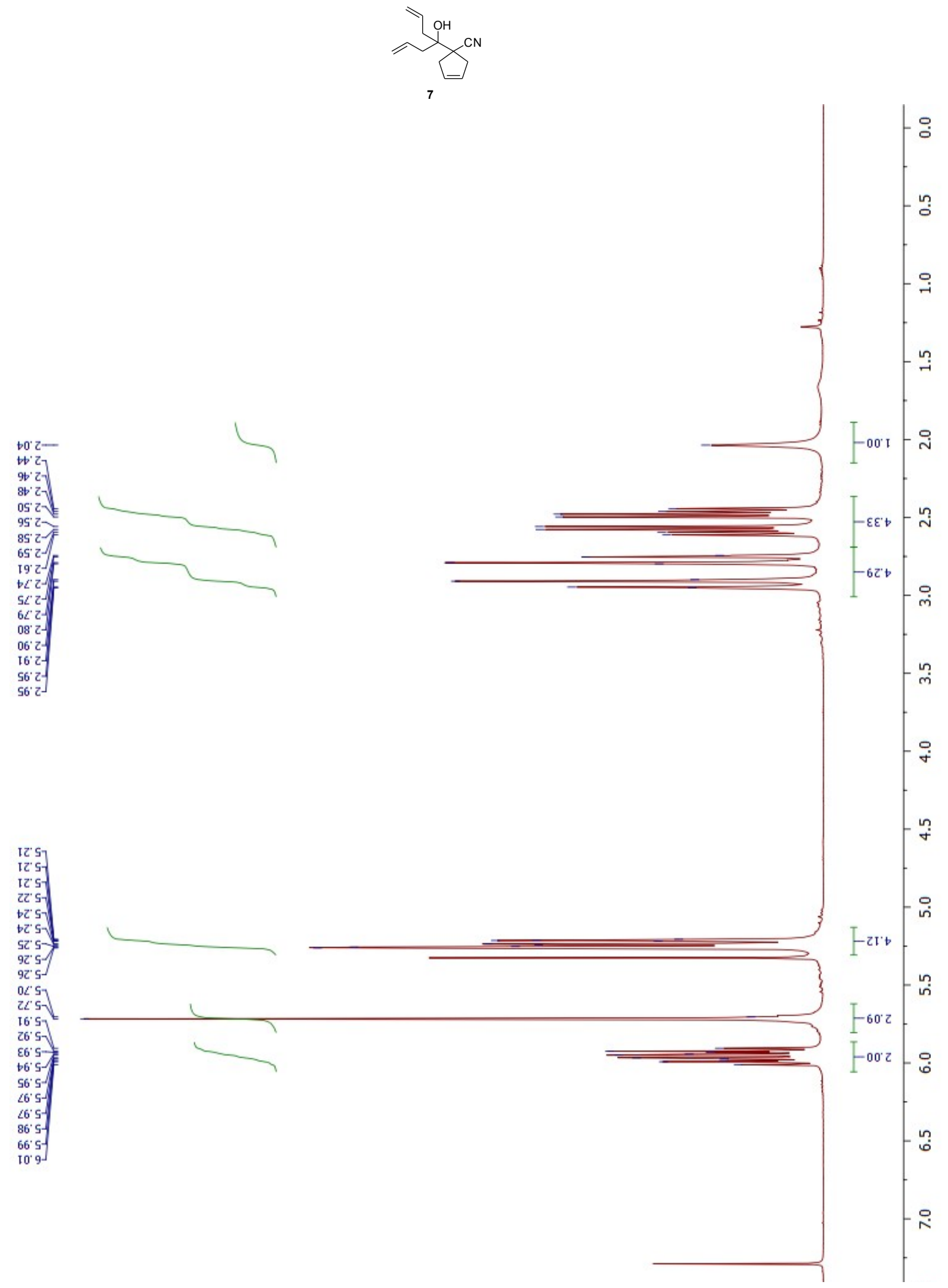


${ }^{13} \mathbf{C}$ NMR $\left(\mathbf{C D C l}_{3}, \quad 100\right.$ MHz) 1-(4-Hydroxyhepta-1,6-dien-4-yl)cyclopent-3-ene-1carbonitrile 7 .
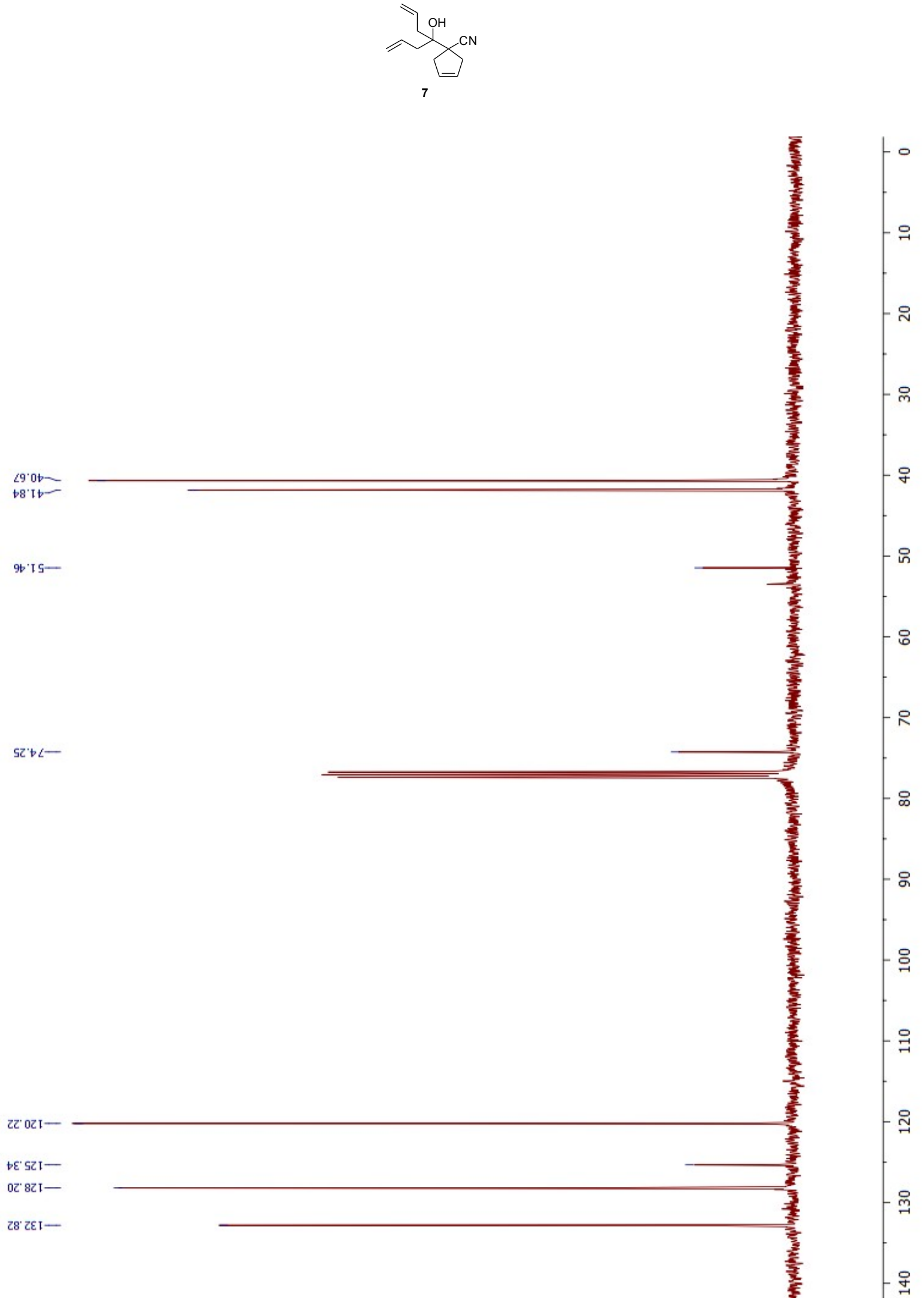
${ }^{1}$ H NMR (acetone- $\boldsymbol{d}_{6}, 400 \mathrm{MHz}$ ) 1'-Hydroxy-[1,1'-bi(cyclopentane)]-3,3'-diene-1-carbonitrile 10.

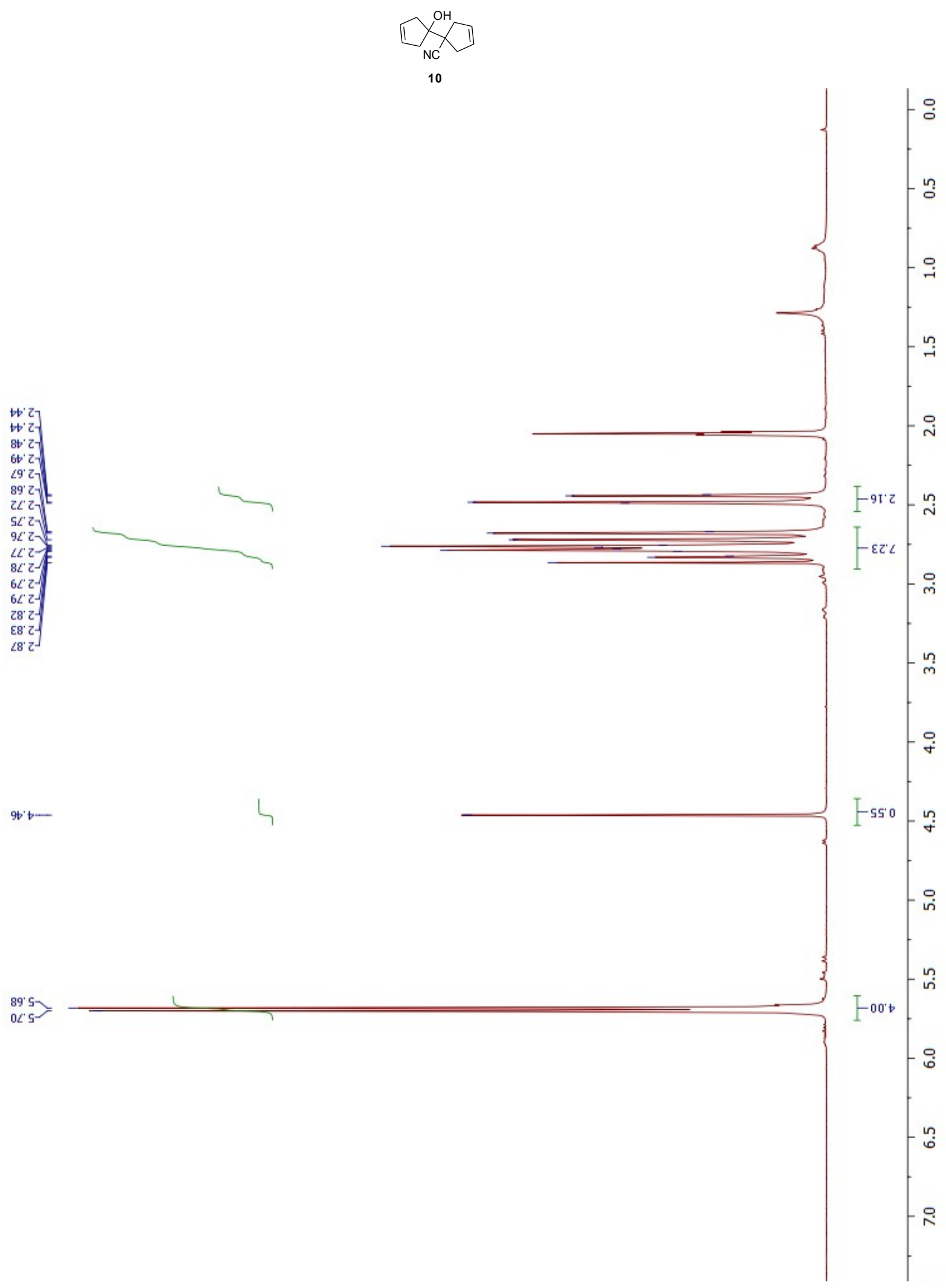


${ }^{13} \mathrm{C}$ NMR (acetone- $d_{6}, 125$ MHz) 1'-Hydroxy-[1,1'-bi(cyclopentane)]-3,3'-diene-1carbonitrile $\mathbf{1 0 .}$

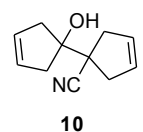

$6 \mathrm{It}^{-}$
$\mathrm{CS}-$

$\angle S$
$D C O S$

$09 \cdot 28-$

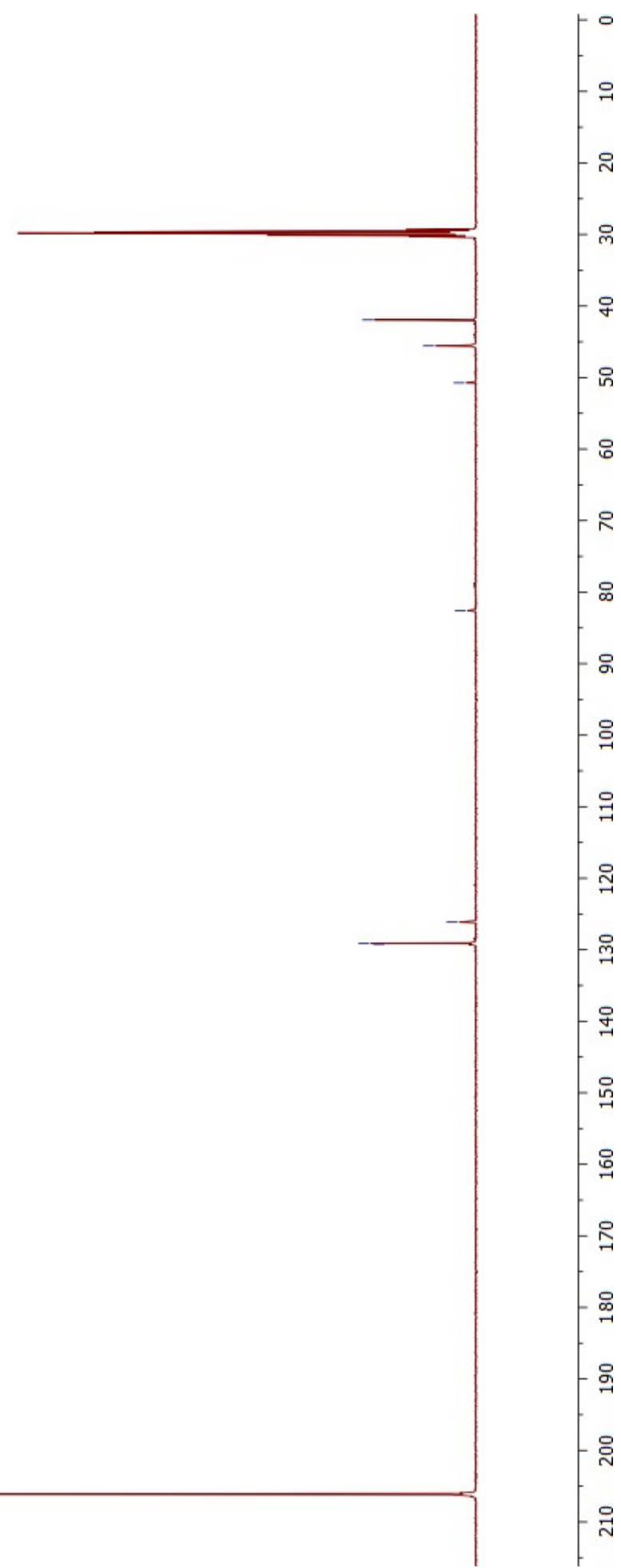


${ }^{1}$ H- ${ }^{1} H$ TOCSY-NMR (acetone- $\boldsymbol{d}_{6}, 500 \mathrm{MHz}$ ) 1'-Hydroxy-[1,1'-bi(cyclopentane)]-3,3'-diene1-carbonitrile $\mathbf{1 0 .}$

Irradiation/excitation of one $=\mathrm{CH}$ alkene environment results in no observed transfer to the other $=\mathrm{CH}$ alkene environment. Transfer to one $\mathrm{CH}_{2}$ environment is observed.

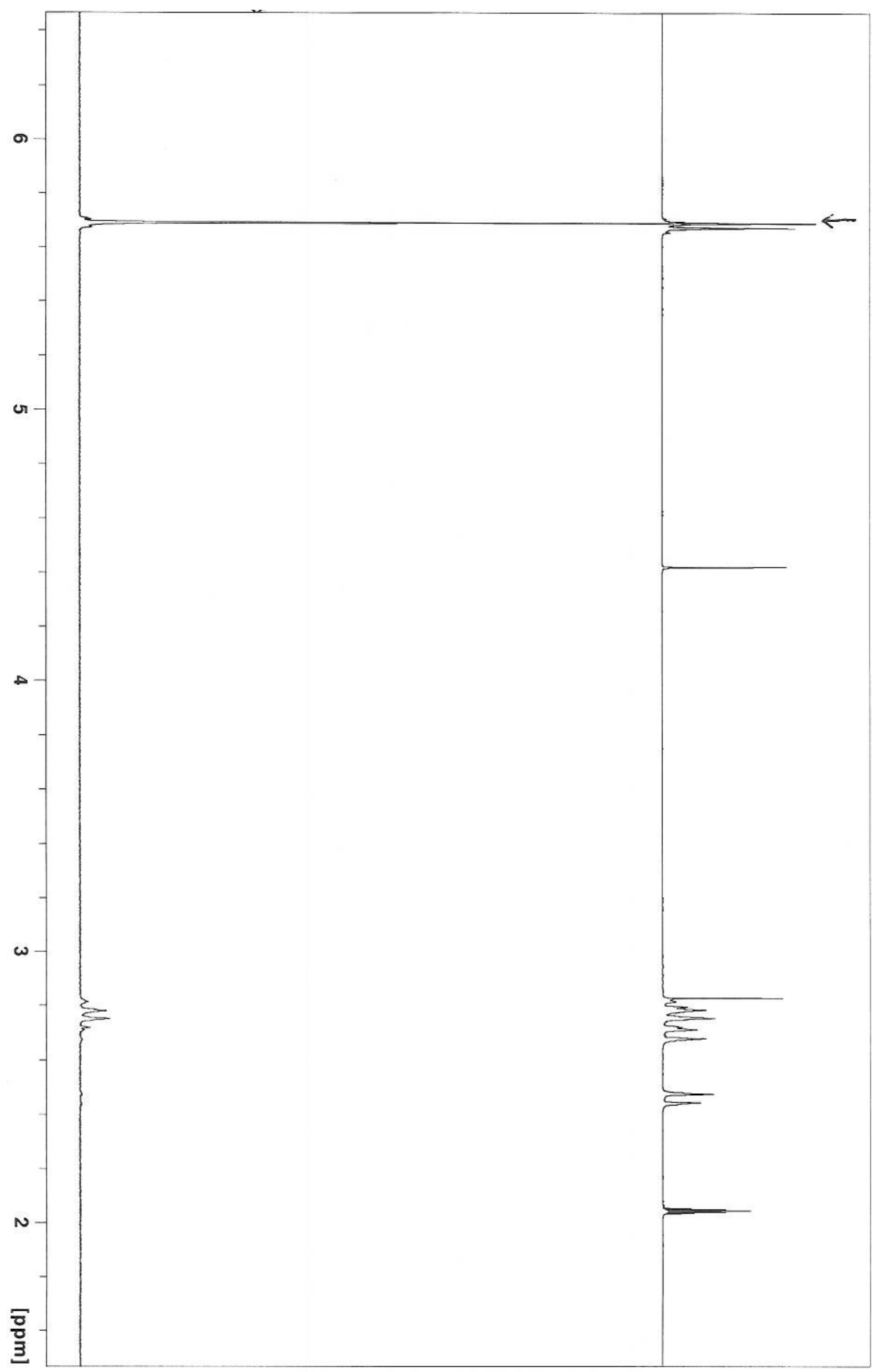


${ }^{1} \mathbf{H}^{1}{ }^{1}$ H TOCSY-NMR (acetone- $\left.\boldsymbol{d}_{6}, 500 \mathrm{MHz}\right)$ 1'-Hydroxy-[1,1'-bi(cyclopentane)]-3,3'-diene1-carbonitrile $\mathbf{1 0 .}$

Complimentary experiment to the previous one.

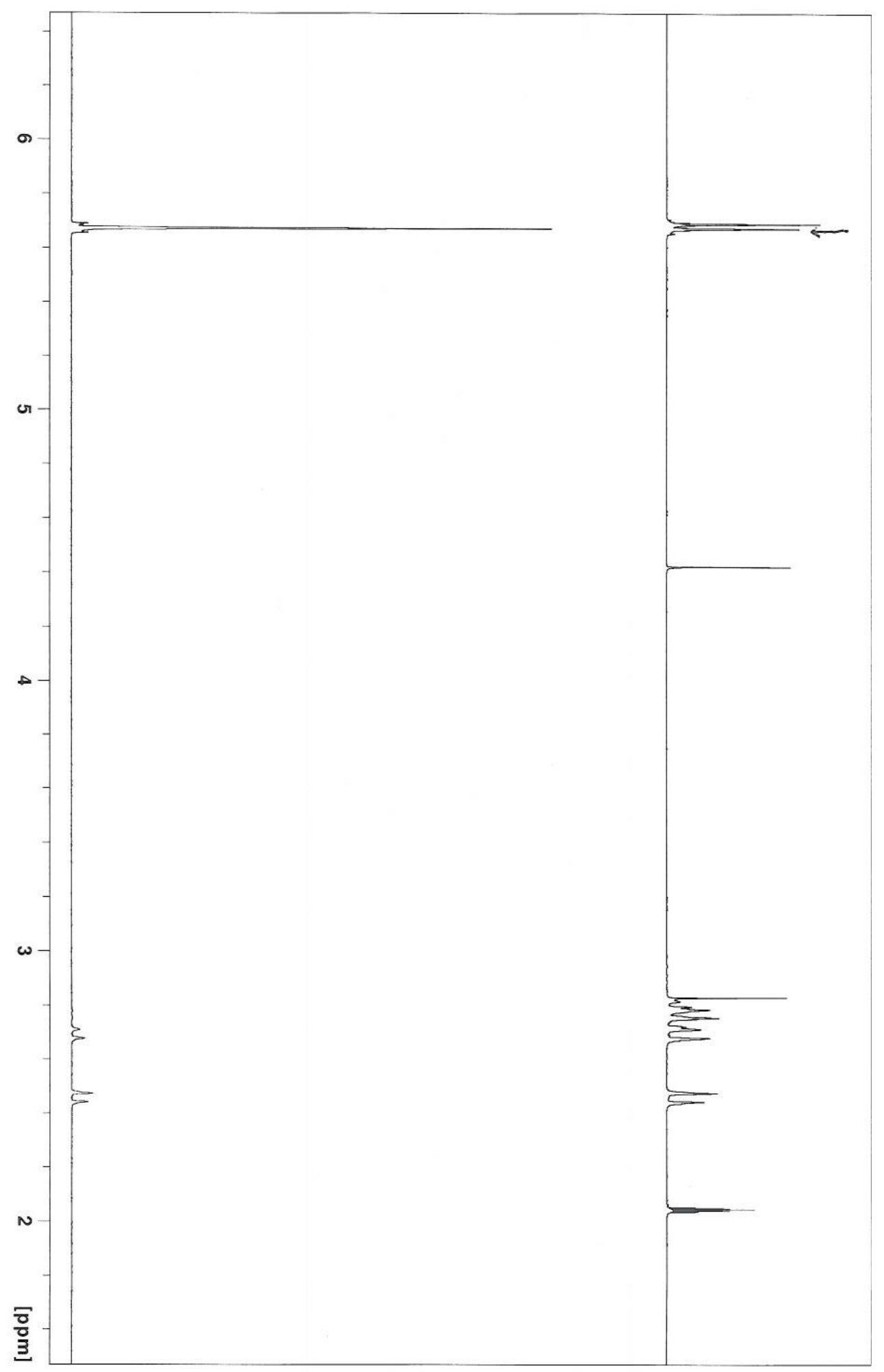




\section{References}

(1) Becke, A. D. Physical Review A 1988, 38, 3098.

(2) Lee, C.; Yang, W.; Parr, R. G. Physical Review B 1988, 37, 785.

(3) Hehre, W. J. R., L.; Schleyer, P. v. R.; Pople, J. A. Ab Initio Molecular Orbital Theory; Wiley: New York, 1986.

(4) Gauss, J. J. Chem. Phys. 1993, 99, 3629.

(5) Lamberto, M.; Kilburn, J. D. Tetrahedron Letters 2008, 49, 6364.

(6) Çetinkaya, B.; Demir, S.; Özdemir, I.; Toupet, L.; Sémeril, D.; Bruneau, C.; Dixneuf, P. H. Chem. - Eur. J. 2003, 9, 2323.

(7) Ding, Z.; Tufariello, J. J. Synth. Commun. 1990, 20, 227. 